\title{
Additional experimental confirmations of Santilli's IsoRedShift and the consequential expected absence of the universe expansion
}

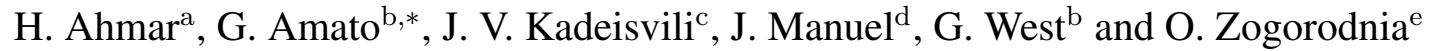 \\ ${ }^{a}$ Institute for UltraFast Spectroscopy and Laser, City College of New York, New York, USA \\ ${ }^{\mathrm{b}}$ Magnegas Corporation, Tarpon Springs, FL, USA \\ ${ }^{\mathrm{c}}$ The Institute for Basic Research, Palm Harbor, FL, USA \\ ${ }^{\mathrm{d}}$ SciRen.Org, 3701 Preakness Place No. 1503 Palm Harbor, FL, USA \\ ${ }^{\mathrm{e}}$ Shevchenko Kyiv National University, Kiev, Ukraine
}

\begin{abstract}
In this paper, we outline the mathematical and physical foundation of the 1991 hypothesis by R. M. Santilli of IsoRedShift (IRS), namely, a frequency shift of light toward the red characterized by the loss of energy by light to a cold medium without any relative motion between the source, the medium and the observer; we outline the corresponding foundations of Santilli's IsoBlueShift (IBS), namely, a frequency shift of light toward the blue characterized by the acquisition of energy by light from a hot medium without relative motion; we show the compatibility of Santilli's IRS and IBS with the axioms of special relativity under their proper mathematical formulations; we review the original experimental confirmations of IRS by Santilli in 2010 for a blue laser light in a tube containing air at pressure; we review the experimental confirmations of both IRS and IBS by G. West and G. Amato in 2011; we review the confirmatory measurements by Santilli, West and Amato done in 2012 on the West coast of Florida on the IRS origin of the redness of the Sun in the transition from the Zenith to the horizon; we present, for the first time, additional confirmatory measurements of IRS of Sunlight from the Zenith to Sunset done by the authors at the island of Kos, Greece, on September 20, 2012; we present, also for the first time, additional confirmatory measurements of IRS of Sunlight from Sunrise to the Zenith done in Cocoa Beach, East Coast of Florida, on October 20, 2012; we review the compatibility of Santilli's 1991 IRS hypothesis with Zwicky's 1929 hypothesis of Tired Light and identify their difference in the process originating the redshift; we recall the fit of cosmological redshift done by P. LaViolette in 1986 with Zwicky's Tired Light hypothesis; we confirm Santilli's 2010 argument according to which the IRS origin of the redness of the Sun at the horizon without relative motion is visual evidence on the expected absence of the expansion of the universe and of related conjectures; and we present the dismissal of various objections against Santilli's IRS and its interpretation of the cosmological redshift. In essence, we agree with Santilli that cosmologists should follow Galileo's teaching by establishing cosmological models via experiments on Earth prior to their application to the universe.
\end{abstract}

Keywords: PACS 42.68.-w, 98.62.Py, 96.10.+i

\section{Mathematical background}

The quantitative treatment of the spectroscopic measurements presented in this paper is based on a broader mathematics developed by the Italian-American scientist Ruggero Maria Santilli when he was

\footnotetext{
${ }^{*}$ Corresponding author: G. Amato, Magnegas Corporation, 150 Rainville Rd, Tarpon Springs, FL 34689, USA. E-mail: research@magnegas.com.
}

1472-7978/13/\$27.50 (c) 2013 - IOS Press and the authors. All rights reserved

This article is published online with Open Access and distributed under the terms of the Creative Commons Attribution NonCommercial License. 
at MIT (from 1974 to 1978), at Harvard University (from 1978 to 1981) and at the Institute for Basic Research (from 1982 on) for the time invariant representation of interior dynamical systems, namely, for extended (thus deformable) particles and electromagnetic waves propagating within generally inhomogeneous and anisotropic physical media. By contrast, Santilli assumes conventional 20th century applied mathematics as being exactly valid for exterior dynamical systems referred to point-like particles and electromagnetic waves propagating in the homogeneous and isotropic vacuum. ${ }^{1}$

A primary difference between interior and exterior systems is the appearance in the form of contact, non-linear, non-local and non-potential interactions that are beyond any hope of consistent representation via a Lagrangian or a Hamiltonian. Santilli's central idea is the representation of said interactions with a generalization $\hat{I}$ of the basic unit $I$ of conventional theories, under the sole condition that $\hat{I}$ is positive-definite, thus admitting the inverse $\hat{I}=1 / \hat{T}$, but otherwise admitting an arbitrary functional dependence on local quantities, such as time $t$, coordinates $r$, velocities $v$, energy $e$, density $d$, frequency $\omega$, temperature $\tau$, and other variables (see the original memoir [1] and the systematic treatment in monographs [2]):

$$
I \rightarrow \hat{I}=\hat{I}(t, r, v, e, d, \omega, \tau, \ldots)=1 / \hat{T}(t, r, v, d, \omega, \tau, \ldots)>0 .
$$

Santilli selected a generalization of the unit for the representation of non-linear, non-local and nonHamiltonian forces because the unity is the basic invariant of any theory. Therefore, the unit is the only known quantity, besides the Hamiltonian, to assure time invariance, that is, the prediction of the same numerical values under the same conditions but at different times. Consequently, the spectroscopic measurements presented in this paper are indeed invariant over time, a property generally violated by other formulations of inhomogeneous and anisotropic theories that, as such, are herein ignored.

Jointly with broadening Eq. (1), Santilli lifted the conventional associative product $A B$ between arbitrary quantities $A$ and $B$ (such as numbers, functions, matrices, operators, etc.) into the form [1,2]

$$
A B \rightarrow A \hat{\times} B=A \hat{T} B,
$$

where $\hat{T}$ remains fixed, under which $\hat{I}$ is indeed to the right and left unit of the theory,

$$
\hat{I} \hat{\times} A=A \hat{\times} \hat{I} \equiv A,
$$

for all elements $A$ of the set considered.

Santilli called the above liftings isotopic in the Greek sense of being axiom-preserving. In fact, $\hat{I}$ is positive-definite as it is the case for $I$, and generalized product Eq. (2) remains associative.

Today, generalized unit Eq. (1) is called Santilli isounit; its inverse $\hat{T}$ is called the isotopic element; and product Eq. (2) is called Santilli isoproduct.

Needless to say, the lifting of the basic unit Eq. (1) and of the related associative product Eq. (2) used for exterior dynamical systems required the corresponding compatible lifting of all applied mathematics used in 20th century physics, including numbers, functional analysis, differential calculus, vector and metric spaces, symmetries, etc. that have been mostly developed by Santilli (see, e.g., the mathematical memoir [3]). The resulting axiom-preserving covering of 20th century applied mathematics is today called Santilli isomathematics, and refers to any mathematics admitting the positive-definite generalized unit $\hat{I}$ at all levels.

\footnotetext{
${ }^{1}$ A presentation of the research conducted at MIT and at Harvard University has been provided by Santilli in Lectures IIA and IIB, level II, of the World Lecture Series available from the link http://www.world-lecture-series.org/level-ii.
} 


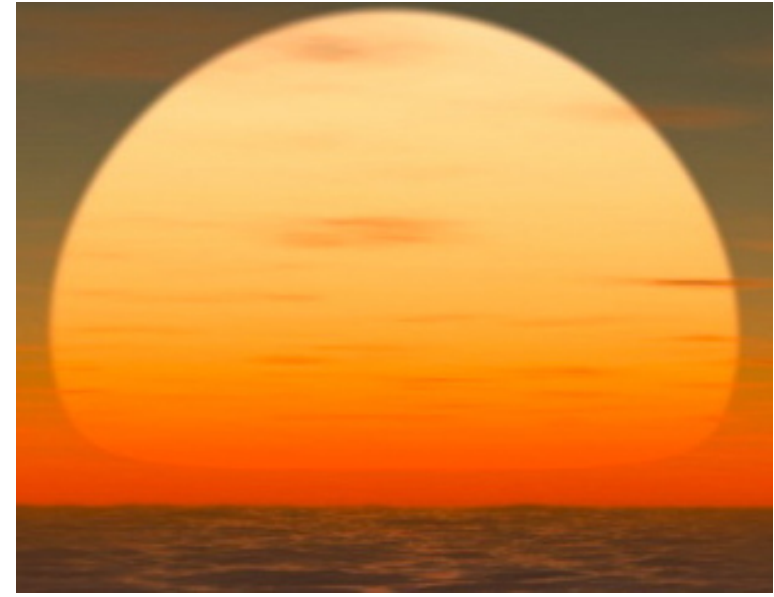

Fig. 1. The main objective of the measurements presented in this paper is to provide experimental confirmation of R. M. Santilli's hypothesis that the redness of "direct" Sunlight at Sunset, as illustrated in the above picture, is primarily due to loss of energy by light to our atmosphere, while scattering is primarily responsible for the decrease of the intensity of Sunlight at the horizon $[3,7]$. Since the Sun redshift at the horizon occurs without appreciable relative motion between the Sun, our atmosphere and the observer (see Section 6 for details), the measurements presented in this paper appear to confirm Santilli's view that the redness of the Sun at the horizon is evidence visible to the naked eye on the lack of expansion of the universe and of related conjectures [9].

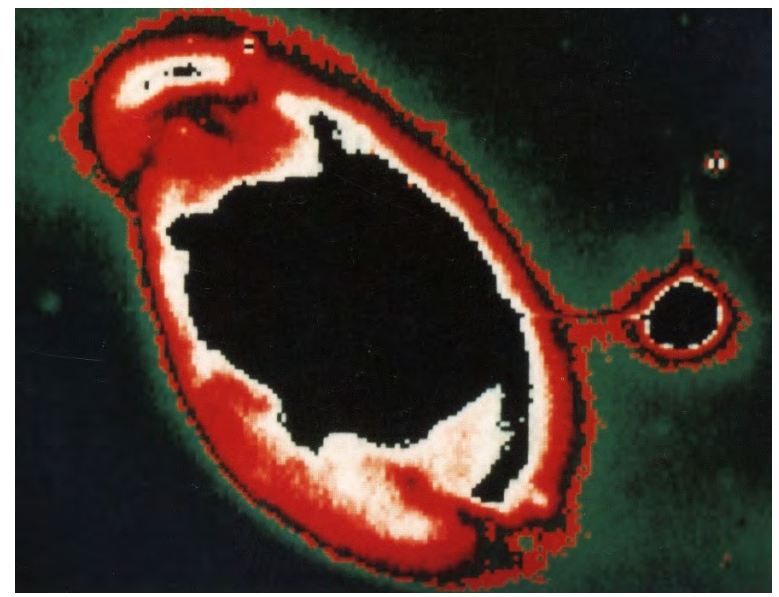

Fig. 2. A picture of a quasar physically connected to a galaxy according to gamma spectroscopic evidence discovered in the early 1970 s by Arp [8], yet their cosmological redshifts are dramatically different. Santilli used this astrophysical evidence in his 1991 monograph [7b] as confirmation that largely different physical media, such as the quasars chromospheres and the innergalactic media, cause corresponding dramatically different loss of energy by light to the medium. According to this view, light exits the quasar and the associated galaxy with dramatically different redshifts. The spectroscopic measurements presented in this paper appear to confirm Santilli's interpretation of Arp's pairs of quasars and their associated galaxies.

Already in the original proposal of 1978 [1], Santilli achieved a step by step isotopic generalization of most of 20th century applied mathematics, including in particular the isotopies of the various branches of Lie's theory. These advances led to a generalization of Lie's algebras with closure rules $[1,2]$

$$
\left[X_{i}, X_{j}\right]=X_{i} \hat{T} X_{j}-X_{j} \hat{T} X_{i}=C_{i j}^{k} X_{k}
$$

today known as the Lie-Santilli isoalgebras, and the corresponding isotopies of Lie's transformation groups, e.g., for the time evolution $[1,2]$

$$
A(t)=\left[e^{H \hat{T} t i}\right] A(0)\left[e^{-i t \hat{T} H}\right]
$$

today known as the Lie-Santilli isogroups. The resulting generalization of Lie's theory is then called the Lie-Santilli isotheory (see, e.g., independent treatment [4]). The importance of the covering isotheory is that, while Lie's theory can solely treat linear, local and Hamiltonian systems, the covering Lie-Santilli isotheory can indeed treat non-linear, non-local and non-Hamiltonian systems since conventional potential interactions are represented via the conventional Hamiltonian $H$ and all non-Hamiltonian interactions are represented with Santilli's isounit $\hat{I}$.

The so-called regular branch of Santilli's isomathematics can now be constructed via the following simple procedure. Note that, by central assumption, interior dynamical systems are not entirely representable with a Hamiltonian. Consequently, the applicable theories must be non-canonical at the classical level and non-unitary at the operator level. One can then subject the conventional unit and product to the non-canonical or non-unitary transform representing the desired non-Hamiltonian interactions, resulting 


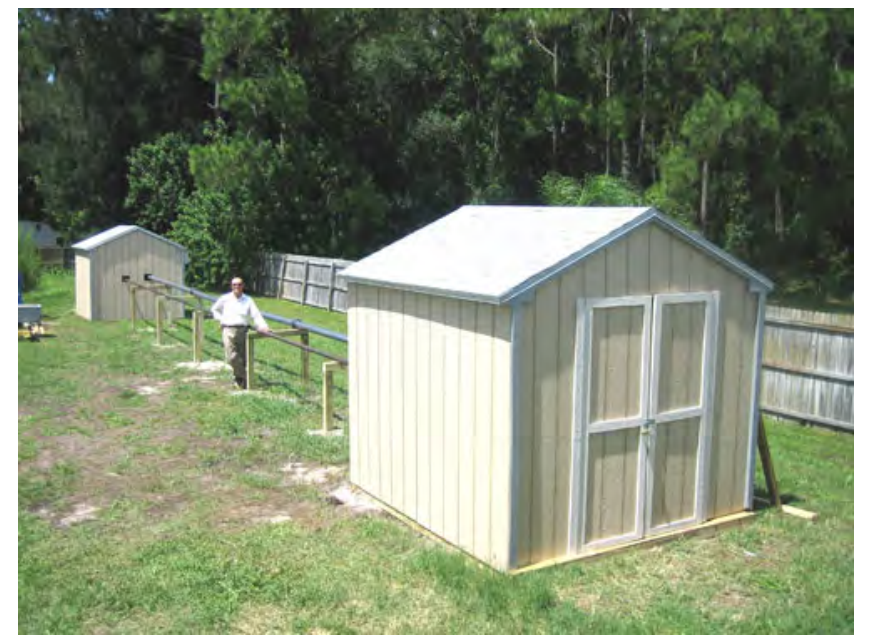

Fig. 3. View of the IsoShift Testing Station built by Santilli at the laboratory of the Institute for Basic Research, Tarpon Springs, Florida, in early 2009.

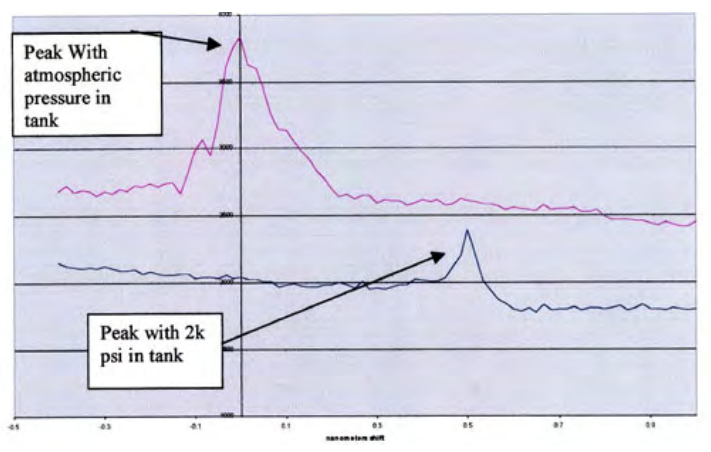

Fig. 4. The first experimental confirmation of Santilli IsoRedShift for blue laser light obtained at the IsoShift Testing Station of Fig. 1 on June 27, 2009 [9].

in this way in Santilli's isounit and isoproduct

$$
\begin{aligned}
& I \rightarrow U I U^{\dagger}=\hat{I} \neq I \\
& A B \rightarrow U(A B) U^{\dagger}=A^{\prime} \hat{T} B^{\prime}, A^{\prime}=U A U^{\dagger}, B^{\prime}=U B U^{\dagger}
\end{aligned}
$$

Consequently, the same transform must be applied, for evident conditions of consistency, to the totality of the remaining mathematics used for exterior dynamical systems, resulting in the novel isonumbers $\hat{n}=n \hat{I}$, isofunctions $\hat{f}(\hat{r})=f(r \hat{I}) \hat{I}$, isodifferentials $\hat{d} \hat{r}=\hat{T} d(r \hat{I})$, etc. (see mathematical memoir [3] for details).

Santilli's central mathematical tool underlying the spectroscopic measurements presented in this paper is given by the axiom-preserving isotopic generalization of the conventional Minkowskian space for the vacuum into a covering geometry capable of representing inhomogeneous and anisotropic media while admitting the conventional Minkowski space as a particular case.

Let $M(x, \eta, I)$ be the conventional Minkowski space with coordinates $x=\left(x^{1}, x^{2}, x^{3}, x^{4}=c t\right)$ (where $c$ is the speed of light in vacuum), metric $\eta=\operatorname{Diag} .(1,1,1,-1)$. unit $I=\operatorname{Diag}(1,1,1,1)$ and historical spacetime line element

$$
x^{2}=x_{1}^{2}+x_{2}^{2}+x_{3}^{2}-t^{2} c^{2},
$$

The Minkowski-Santilli isospace [5] is characterized by $\hat{M}(\hat{x}, \hat{\eta}, \hat{I})$ with coordinates $\hat{x}=x \hat{I}$, isometric $\hat{\eta}=\hat{T} \eta$ and isounit $\hat{I}=1 / \hat{T}$. Under the assumption that the isospace is not singular and symmetric, its isometric, isotopic element and isounit can always be diagonalized into the form

$$
\begin{aligned}
& \hat{\eta}=\hat{T} \eta=\left(\hat{T}_{\alpha}^{\beta}(t, r, v, e, d, \omega, \tau, \ldots) \eta_{\beta \gamma}\right), \\
& \hat{T}=\operatorname{Diag} \cdot\left(1 / n_{1}^{2}, 1 / n_{2}^{2}, 1 / n_{3}^{2}, 1 / n_{4}^{2}\right)>0, \\
& \hat{I}=\operatorname{Diag} \cdot\left(n_{1}^{2}, n_{2}^{2}, n_{3}^{2}, n_{4}^{2}\right)=1 / \hat{T}>0, n_{\mu}=n_{\mu}(t, r, v, e, d, \omega, \tau, \ldots)>0, \mu=1,2,3,4,
\end{aligned}
$$


resulting in the most general possible non-singular and symmetric spacetime line element [6]

$$
\hat{x}^{\hat{2}}=\left(\frac{x_{1}^{2}}{n_{1}^{2}}+\frac{x_{2}^{2}}{n_{2}^{2}}+\frac{x_{3}^{2}}{n_{3}^{2}}-t^{2} \frac{c^{2}}{n_{4}^{2}}\right) \hat{I},
$$

which contains as particular cases all possible spacetimes in (3+1)-dimension (such as the Minkowskian, Riemannian, Finslerian and other spacetimes), where: the $n$ 's are called the characteristic quantities of the medium considered; $n_{4}$ is the conventional index of refraction providing a geometrization of the density of the medium normalized to the value for the vacuum $n_{4}=1 ; n_{1}, n_{2}, n_{3}$ provide a geometrization of the medium considered normalized to the values for the vacuum $n_{1}=n_{2}, n_{3}=1$; the inhomogeneity of the medium considered is represented by the dependence of the characteristic quantity in the local variables (e.g., in the elevation for the case of Earth's atmosphere); and the anisotropy of the medium in a given direction $s$ is represented by different values $n_{4} \neq n_{s}$.

A number of authors have proved that the Minkowski-Santilli isogeometry is "directly universal" for all possible non-singular and symmetric spacetimes, namely, said geometry is capable of representing all possible spacetimes of the class admitted (universality) in the frame of the observer (direct universality) [5].

Santilli's central hypothesis subjected to experimental verifications in this paper is that the presence of matter (or of energy) alters the Minkowskian geometry of the vacuum into the iso-Minkowskian form. As we shall see, this basic hypothesis is not only plausible, but appears to be experimentally verifiable.

\section{Theoretical background}

Following the prior construction of the needed isomathematics, the theoretical research underlying this paper was initiated by Santilli with the solution in 1983 [6] of the historical Lorentz problem, namely, the achievement of the universal invariance of all infinitely possible, locally varying speeds of electromagnetic waves within physical media (including infrared, radio and other waves for which the reduction to photons is not consistently possible)

$$
C=\frac{c}{n(t, r, v, e, d, \omega, \tau, \ldots)} .
$$

As it is well known, Lorentz achieves the solution of the problem for the case of constant speed $c$, resulting in the historical invariance of the Minkowskian spacetime Eq. (7) which is at the foundation of special relativity

$$
\begin{aligned}
& x^{\prime 1}=x^{1}, x^{\prime 2}=x^{2}, \\
& x^{\prime 3}=\gamma\left(x^{3}-\beta x^{4}\right), \\
& x^{\prime 4}=\hat{\gamma}\left(x^{4}-\beta x^{3}\right), \\
& \beta=\frac{v}{c}, \gamma=\frac{1}{\sqrt{1-\beta^{2}}} .
\end{aligned}
$$

However, Lorentz failed to achieve the invariance of the locally varying speeds Eq. (10). Santilli recognized that this was due to the insufficiency of the basic mathematics used to construct the invariance, Lie's theory, due to its strictly linear, local and Hamiltonian character, while the problem considered is strictly non-linear, non-local and non-Hamiltonian. 


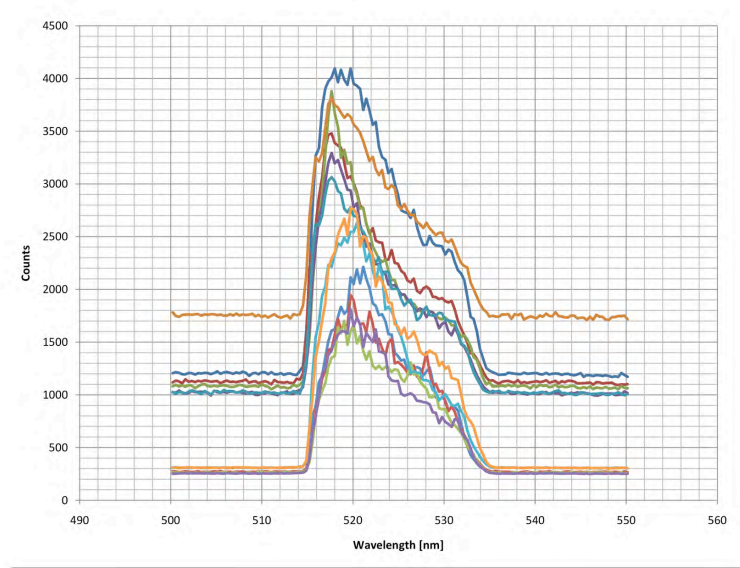

Fig. 5. The first experimental confirmation of the increase of IsoRedShift with the increase of the pressure or, equivalently, the increase of the density first obtained by Santilli at the IsoShift Testing Station of Fig. 1 in July 2009 [9]. Said increase of the IRS with the increase of the density has crucial relevance for prorating the measurements in our atmosphere to the intergalactic medium (see Section 9 for details).

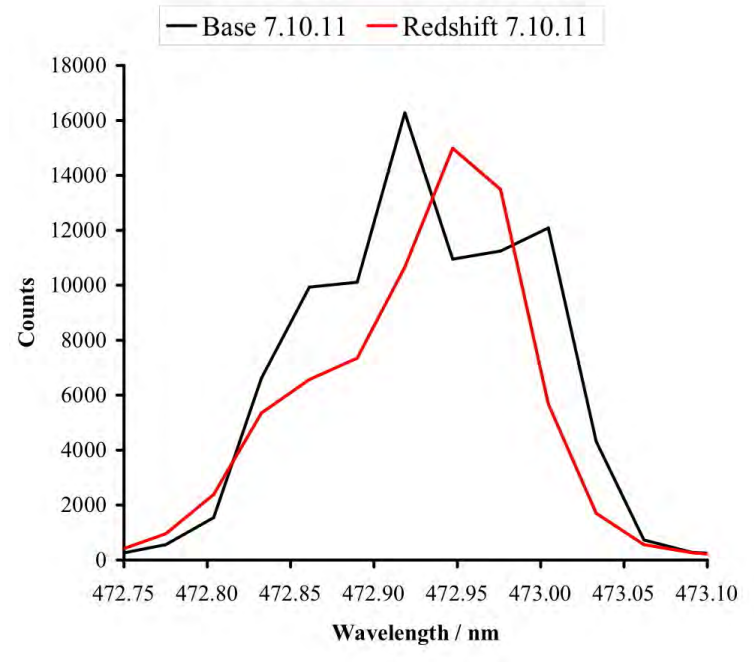

Fig. 6. One of the systematic experimental confirmations obtained by West and Amato [12] on the existence of Santilli's IRS via the same IsoShift Testing Station used in Ref. [9] but at temperature below $70^{\circ} \mathrm{F}$. In this scan, the peak to the left denotes the blue laser light under vacuum and that to the right represents the same light traversing air at 2,000 psi without any relative motion between the source, the pipe and the wavelength analyzer.

For this reason, Santilli first constructed the isotopies of Lie's theory [1,2], and then solved the historical Lorentz problem in one page of the letter paper [6] via the identification of the isotopic element $\hat{T}$ in the invariance Eq. (9) and then the use of the Lie-Santilli isogroups, such as in Eq. (5). This procedure yielded the universal symmetry for all infinitely possible iso-Minkowskian line element Eq. (9) which we write in the form

$$
\begin{aligned}
& x^{61}=x^{1}, x^{62}=x^{2}, \\
& x^{63}=\hat{\gamma}\left(x^{3}-\hat{\beta} \frac{n_{3}}{n_{4}} x^{4}\right), \\
& x^{\natural 4}=\hat{\gamma}\left(x^{4}-\hat{\beta} \frac{n_{4}}{n_{3}} x^{3}\right), \\
& \hat{\beta}=\frac{v_{3} / n_{3}}{c_{o} / n_{4}}, \hat{\gamma}=\frac{1}{\sqrt{1-\hat{\beta}^{2}}} .
\end{aligned}
$$

now called the Lorentz-Santilli transformations. Subsequently, Santilli studied the isotopies of each individual aspect of the Lorentz-Poincaré (LP) symmetry (whose review is here omitted for brevity), resulting in a covering symmetry now called the Lorentz-Poincaré-Santilli (LPS) isosymmetry. ${ }^{2}$

\footnotetext{
${ }^{2}$ Santilli's papers on the isotopies of the various aspects of the Lorentz-Poincaré symmetry, as well as of its spinorial covering, are available in free pdf download at http://www.santilli-foundation.org/LPS-references.php.
} 
By the early 1990s, the axiom-preserving isotopies of all the essential aspects of special relativity were well identified and presented in monographs [7] of 1991. The emerging covering of relativity is today known as Santilli isorelativity, where the prefix "iso" stands to emphasize the preservation of the abstract axioms of special relativity and the mere presentation of a broader realization.

As it is well known, the LP symmetry characterizes uniquely and unambiguously all axioms of special relativity. Along exactly the same lines, the LPS isosymmetry characterizes uniquely and unambiguously all axioms of the covering relativity. The axiom of isorelativity studied in this paper is that describing frequency shifts, which was already identified in paper [6], but studied in detail in Volume [7b], and can be written in first approximation in the selected $s$-space direction (see Ref. [7b] for full treatment)

$$
\omega \approx \omega_{o}\left(1 \pm \frac{v_{s}}{c} \frac{n_{4}}{n_{s}}+\ldots\right)
$$

today known as the Doppler-Santilli IsoShift and referred to a frequency shift partially due to relative motion between the source and the observer, and partially due to the medium in which the electromagnetic waves propagates. Note that the effect depends on the selected space direction $s$ due to the inhomogeneous and anisotropic character of the medium, a feature absent in special relativity due to the homogeneity and isotropy of the vacuum. The reader should keep in mind such a directions dependence of frequency shifts to prevent possible misrepresentations.

In Volume [7b], Santilli clearly identified the fact that the characteristic quantities $n_{\mu}$ have a dependence on velocities. Therefore, isolaw Eq. (13) admits a particular case in which a frequency shift occurs without any relative motion between the source, the medium and the observer,

$$
\omega_{e}=\omega_{0}(1 \pm K d+\ldots), K d=\left[\frac{v^{2}}{c^{2}} \frac{n_{4}}{n_{3}}\right]_{v=0} \neq 0,
$$

where $\omega_{e}\left(\omega_{o}\right)$ is the frequency measured (at the origin) and $d$ represents the distance traveled by light in the medium.

Law Eq. (14) is nowadays known as Santilli IsoShift law, with classification [7b] (see later on Fig. 32 for a conceptual rendering):

1) IsoRedShift (IRS), occurring for the minus sign and representing the loss of energy $E=\hbar \omega$ electromagnetic waves to a medium at sufficiently low temperature;

2) IsoBlueShift (IBS), occurring for the plus sign, and representing the acquisition of energy $E=\hbar \omega$ by electromagnetic waves from a medium when at sufficiently high temperature; and

3) NoIsoShift (NIS), occurring for media at intermediate temperature between the IRS and the IBS, and representing the case when the energy lost by electromagnetic waves to the medium is equal to the energy acquired, resulting in no frequency shift, in which case $K=0$.

In a number of cases, we have the combination of a pure Doppler frequency shift due to relative motion between the source and the observer, plus Santilli IsoShift due to propagation of light within a medium. As we shall see, this is the case of Sunset (Sunrise), in which the observer moves away (toward) from the Sun due to Earth's rotation, plus a loss of energy by light traveling in Earth's atmosphere. In this case, Santilli introduces the realization of the characteristic quantities

$$
\frac{n_{s}}{n_{4}} \approx 1 \pm K^{\prime} d+\ldots
$$

with resulting general expression for the Doppler-Santilli IsoShift

$$
\omega=\omega_{0}\left[1 \pm \frac{v}{c}(1 \pm K d+\ldots)\right]
$$




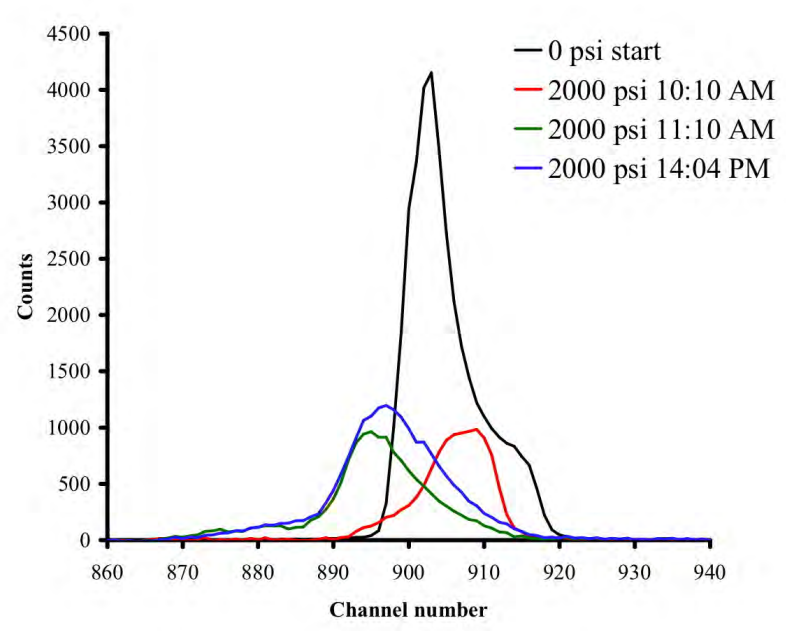

Fig. 7. The first of systematic experimental confirmations obtained by G. West and G. Amato [12] on the existence of Santilli's IBS via the same IsoShift Testing Station used in Ref. [9]. In this scan, the central peak denotes the blue laser light under vacuum; the peak to the right denotes IRS obtained at night; and the peak to the left presents the first measured IBS by mere chance due to the increase of the temperature of the pipe under the strong Florida Sun while continuing the measurements.

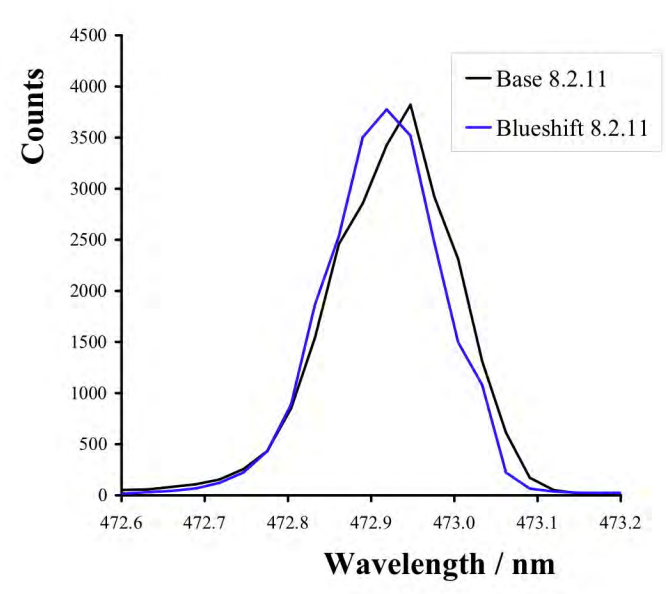

Fig. 8. One of the systematic experimental confirmations obtained by West and Amato [12] on the existence of Santilli's IBS via the same IsoShift Testing Station used in Ref. [9] but operated at over $140^{\circ} F$. In this scan, the peak on the right represents the blue laser light under vacuum, while the peak on the left represents IsoBlueShifted light.

representing all four different combinations of source motion away and toward the observer and light losing to or acquiring energy from the medium.

Santilli proposed the IRS from the visual observation that the redness of the Sun at Sunset and Sunrise occurs without any relative motion between the Sun and Earth. Therefore, Santilli submitted in Ref. [7b], page 330, 1991, the following:

Proposed experiment: Measure in our laboratories the possible Isoredshift of sunlight in the transition from the zenith to the horizon.

Additionally, the IRS was proposed on grounds of information from NASA planetary missions according to which the pitch of radio communications decreases (thus characterizing a redshift) when the spaceship travels behind a planetary atmosphere.

As an additional evidence on the existence of the IRS, Santilli provided in Ref. [7b] a numerical representation of the large differences in cosmological redshift for Arp's quasars when physically connected to galaxies according to clear gamma spectroscopic evidence [8]. Said large differences were interpreted as being merely due to the large differences in size and density between quasar's chromospheres and the innergalactic media, as a result of which light exits the quasars and the connected galaxies already vastly IsoRedShifted (see general review [33] for details).

\section{Compatibility of Santilli's IsoShifts with special relativity}

It is generally believed that the frequency shift occuring without relative motion "violates" Einstein's special relativity. Santilli has proved that this belief is erroneous and it is generally due to a lack of technical knowledge of the field. As evident from the title of Refs. [7], Santilli has developed isomathematics 


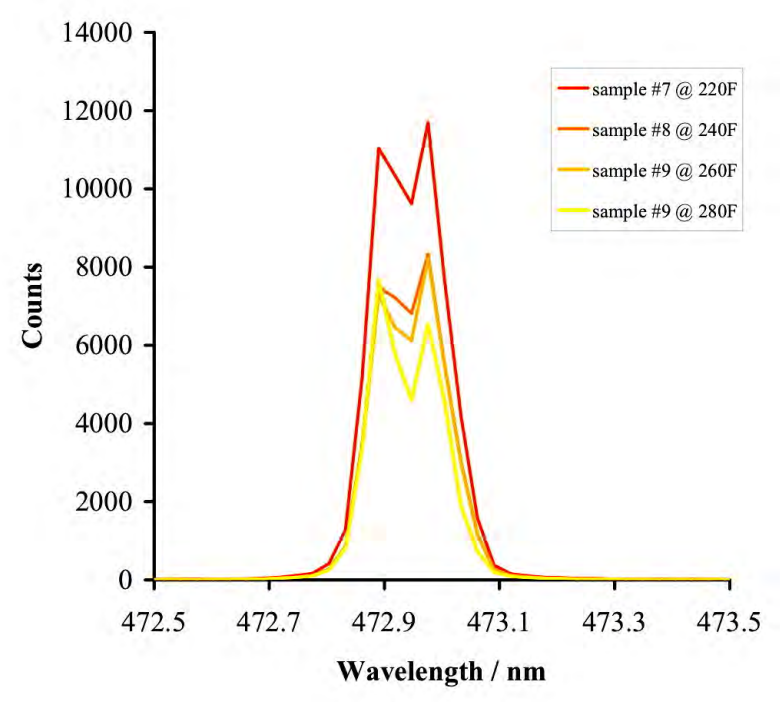

Fig. 9. One of the systematic experimental confirmations obtained by West and Amato [12] on the existence of Santilli's NIS via the same IsoShift Testing Station used in Ref. [9] but operated at temperatures between $70^{\circ} \mathrm{F}$ and $140^{\circ} \mathrm{F}$.

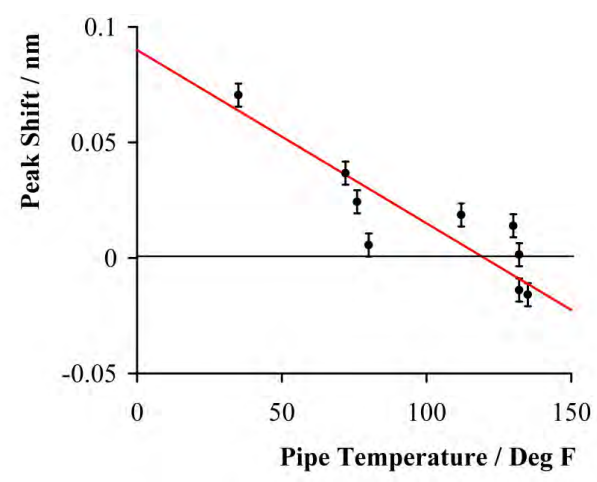

Fig. 10. A plot of some of the measurements of Santilli IRS, IBS, and NIS obtained by G. West and G. Amato via the the same IsoShift Testing Station used in Ref. [9] but operated at temperatures from below $70^{\circ} \mathrm{F}$ to over $140^{\circ} \mathrm{F}$, thus establishing the clear dependence of Santilli IsoShifts in the gas temperature, while the continuous transition from the IRS to the IBS establishes the necessary existence of the NIS.

precisely for the preservation of Einstein's axioms, and the mere presentation of their broader realization for the extension of their arena of applicability to interior dynamical conditions.

Firstly, the Lorentz-Santilli isosymmetry Eq. (3) is locally isomorphic to the conventional Lorentz symmetry to the point of preserving the original structure constants [6,7]. Therefore, no prediction of transforms Eq. (3) can be claimed on technical grounds to violate the Lorentz symmetry and the ensuing special relativity.

Secondly, the Lorentz transforms provide the invariance of the constant speed $c$ without any identification of its numerical value, which is set by physical arguments. Therefore, the Lorentz transforms equally apply for a constant speed $C=c / n_{4}$ and no violation of special relativity can be claimed for constant speeds of light within physical media [7].

Finally, the maximal causal speed in the $s$ direction within physical media represented by the isoline element Eq. (9) is characterized by the light isocone [6,7]

$$
\hat{x}^{\hat{2}}=\frac{x_{s}^{2}}{n_{s}^{2}}-\frac{c^{2}}{n_{4}^{2}}=0,
$$

resulting in the maximal causal value

$$
V_{\max }=c \frac{n_{s}}{n_{4}} \text {. }
$$

The replacement in the conventional Lorentz transforms Eq. (11) of the speed of light $c$ with the expression $V_{\max }$ yields the Lorentz-Santilli transformations Eq. (12) identically,

$$
\begin{aligned}
& x^{61}=x^{1}, x^{62}=x^{2}, \\
& x^{63}=\gamma\left(x^{3}-\beta x^{4}\right), \\
& x^{64}=\hat{\gamma}\left(x^{4}-\beta x^{3}\right),
\end{aligned}
$$




$$
\beta=\frac{v}{V_{\max }}, \gamma=\frac{1}{\sqrt{1-\beta^{2}}} .
$$

In any case, a condition for the correct realization of Santilli's isotopies is that, when represented on isospaces over isofields, light isocone Eq. (17) is the perfect cone with maximal causal speed $c$ as for the original Minkowski space [7b]. This is due to the fact that the cone axes are indeed mutated from their original unit value,

$$
\left(1_{s}, 1_{4}\right) \rightarrow\left(1 / n_{s}^{2}, 1 / n_{4}^{2}\right),
$$

but the relative units are mutated by the inverse amounts,

$$
\left(1_{s}, 1_{4}\right) \rightarrow\left(n_{s}^{2}, n_{4}^{2}\right)
$$

thus preserving the original Minkowskian light cone identically, again, when formulated in a mathematically correct form. Consequently, in the absence of any deformation of the conventional light cone on isospace over isofields, any claim of "violation" of Einstein's special relativity within physical media is scientifically unfounded.

Therefore, the measurements of frequency shift without relative motion presented in this paper should not be considered as "violating" Einstein special relativity, because in reality, said measurements honor Einstein's name by showing the fully broadened representational power of his axioms when treated with Santilli's isomathematics.

It is important to point out that isotopic reformulation Eq. (19), even though formally identical to the conventional Lorentz transformations, carries significant physical implications. In fact, Lorentz transformations leave invariant Minkowski line element which is notoriously quadratic in time thus solely applying for the representation of events reversible over time, such as atomic structures, particles in accelerators, and other systems.

By contrast, isotopic reformulation Eq. (19) leaves invariant line element Eq. (9) which is no longer necessarily quadratic in time since $n_{4}$ can also depend on time, as indicated earlier. Therefore, the Minkowksi-Santilli isogeometry and related isoinvariant Eq. (9) can indeed represent nonconservative and irreversible events, as necessary for a consistent treatment of IsoShifts.

\section{First experimental confirmation of Santilli IsoRedShift}

Since the formulation of the IRS in 1991 [7b], Santilli submitted proposals to various physics laboratories around the world to conduct measurements of light propagating in air at various pressures in order to verify or dismiss the existence of a redshift without relative motion.

Following about two decades of refusals to consider the proposed measurements, despite their low cost and fundamental implications, Santilli had no choice other than that of conducting the needed measurements himself.

Therefore, with the assistance of the technicians at the Institute for Basic Research in Florida, as well as the assistance of external laser experts, Santilli constructed the IsoShift Testing Station shown in Fig. 3 that comprises: an initial air-conditioned cabin containing a blue laser; a second air-conditioned cabin containing wavelength analyzers; the laser and analyzer being interconnected by a $60 \mathrm{ft} \approx 18 \mathrm{~m}$ long steel pressure pipe containing air at the maximal pressures up to 2,000 psi $\approx 137$ bars.

Following several months of measurements, Santilli released the results for publication in Ref. [9] by stressing that the main scope of the measurements was to establish the existence of the IRS, without any 


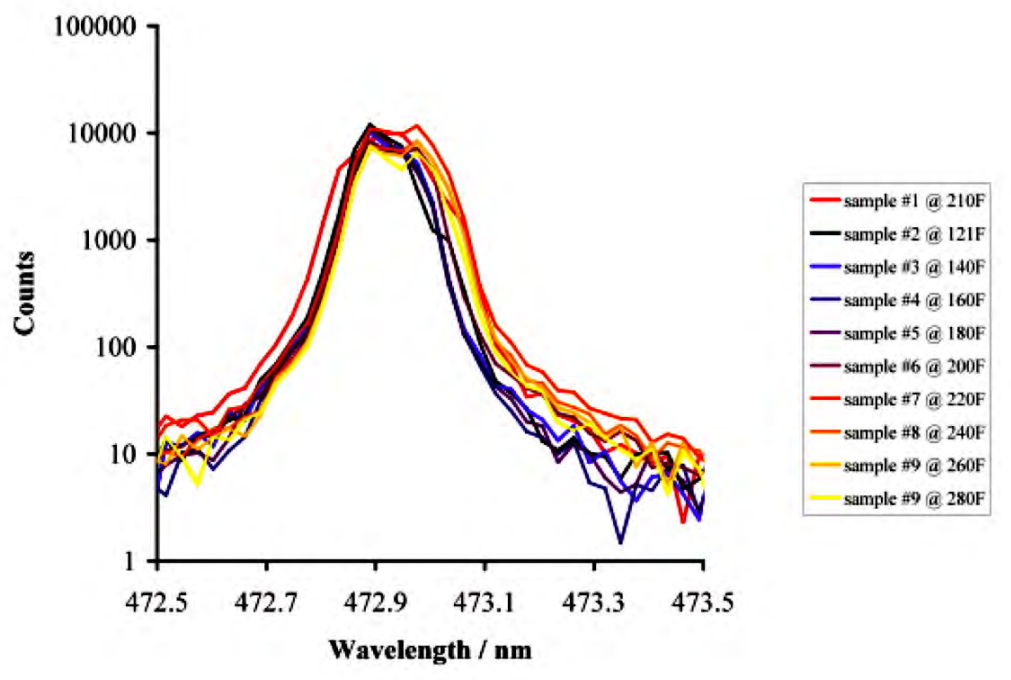

Fig. 11. A plot of some of the measurements of obtained by G. West and G. Amato in the reflection of a blue laser light on a polished metal surface at temperatures increasing close to the melting point, to illustrate the absence of a frequency shift for elastic scattering assumed in Ref. [9].
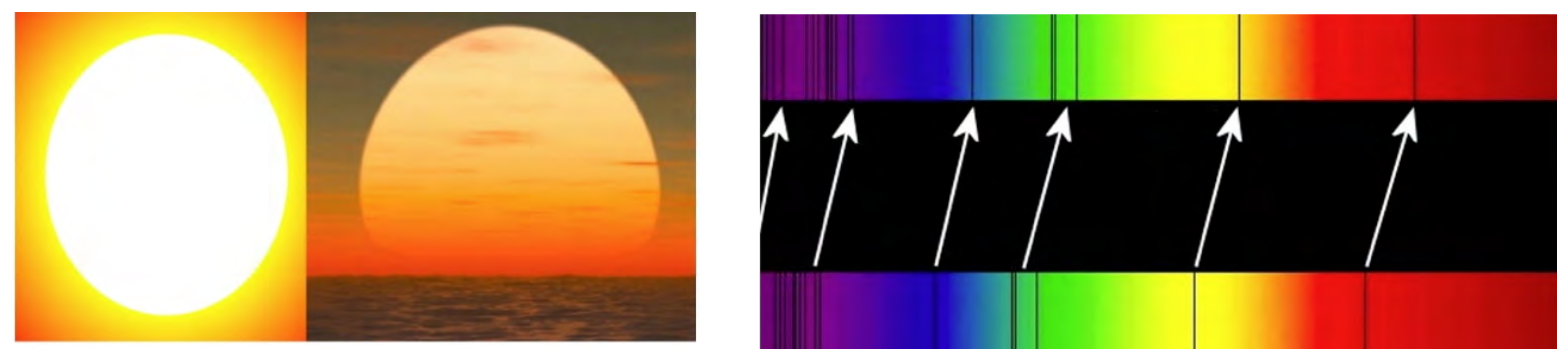

Fig. 12. Following Refs. [9-13], Santilli et al. [14] conducted spectroscopic analyses of the entire spectrum of visible Sunlight in the transition from the Zenith to the horizon (left figures) because it is equivalent to the cosmological redshift of distant galaxies (right view). Therefore, experimental measurements of the Sun redshift have direct relevance for the cosmological redshift.

possibility of conducting statistical averages with the available experimental set up, due to the evident variation of the temperature with pressure, inevitable pressure leaks, and other uncontrollable variations precluding the identification of an exact numerical value of the IRS with related error.

It is evident that scattering of the laser light does occur in the pipe used for measurements [9]. In fact, scattering is responsible for the evident decrease of the intensity on the considered laser light. However, to minimize possible misrepresentations, Ref. [9] stresses that the measured IRS strictly refers to direct blue laser light and not to that scattering in the tube. The latter was carefully avoided via collimators, slits and other means solely permitting the entrance in the wavelength analyzer of direct blue laser light, namely, the portion of the laser light propagating along a straight line for which no consistent definition of scattering can apply, thus leaving the loss of energy by the laser light to the air in the tube the sole 

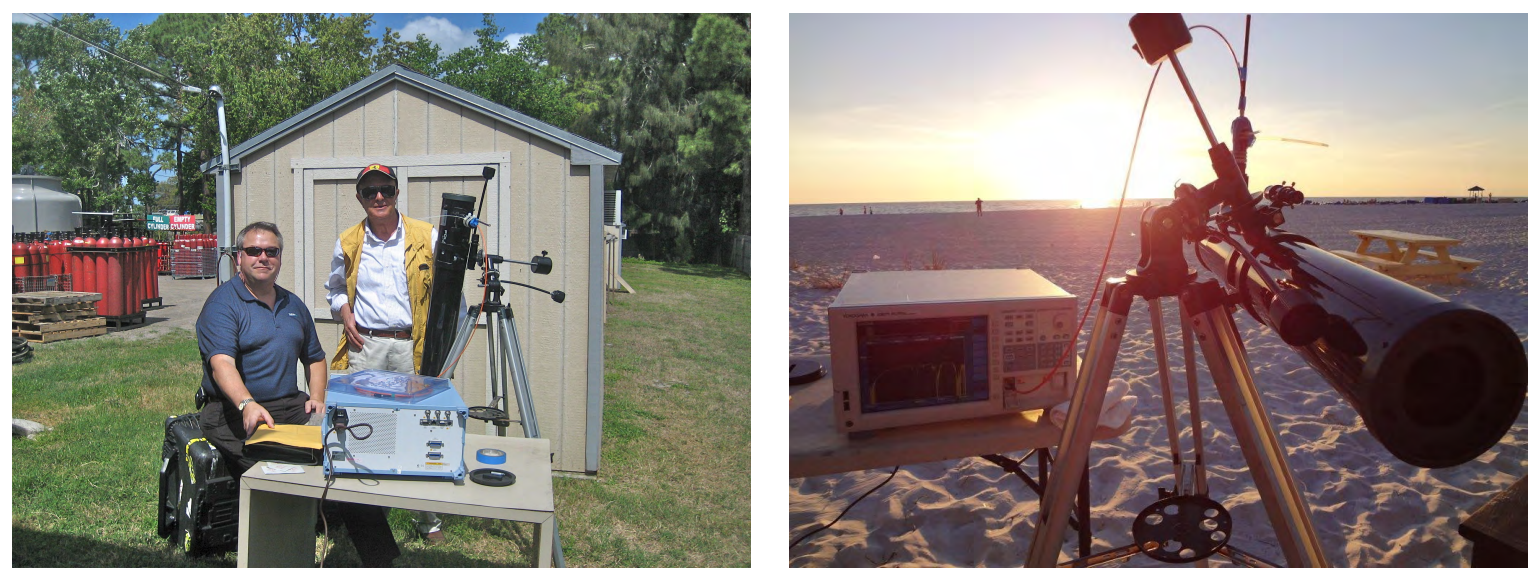

Fig. 13. The experimental set up for the scan of Sunlight from the Zenith to the horizon used in Ref. [14] comprising a conventional refraction telescope with a Yokogawa wavelength analyzer directly connected to the eyepiece. The top picture shows the the calibration of the instrument by a manufacturer representative for scans of the Sun at the Zenith, and the position of the instrument at sunset.

plausible physical origin of the redshift. ${ }^{3}$

Santilli's 2010 paper [9] is the fundamental paper in the field following the original conception [6, 7], since all other papers in this new field [10-14] essentially provide experimental confirmations of the various aspects treated Ref. [9], such as:

1) The existence of a systematic and measurable IRS for blue laser light propagating within $60 \mathrm{ft} \approx$ $18 \mathrm{~m}$ filled up with air in the transition from vacuum to $2,000 \mathrm{psi} \approx 137$ bars and at the winter temperature in Florida of about $70^{\circ} F \approx 21^{\circ} \mathrm{C}$ yielding a frequency shift toward the red of approximately $0.5 \mathrm{~nm}$ without any relative motion between the source, the air and the analyzer (Fig. 4).

2) Systematic measurements of the increases of the IRS of said blue laser light with the increase if the pressure or, equivalently, of the distance traveled by said blue laser light, in air at the indicated temperature (see Fig. 5).

3) Approximate estimates that, when prorated to Sunlight at the horizon, the IRS measured in the pipe can indeed represent the redshift of the Sunlight in the transition from the Zenith to the horizon which is about $100 \mathrm{~nm}$. In fact, the travel of the blue laser light in the tube for $60 \mathrm{ft} \approx 18 \mathrm{~m}$ at 2,000 psi $\approx 137$ bars corresponds to a travel of the same blue laser light in air at atmospheric pressure at sea for about $747 \mathrm{ft} \approx 2,466 \mathrm{~m}$. Similarly, the trajectory of Sunlight at the horizon (thus along the tangent from sea level to the stratosphere) can be approximated as corresponding to about $150,000 \mathrm{ft} \approx 500 \mathrm{~km}$ at atmospheric pressure at sea level. Therefore, the prorating to Sunlight at the horizon of an IRS of $0.5 \mathrm{~nm}$ measured in the pipe (and hereon assumed as occurring at the low temperature of the upper atmosphere) yields indeed the IRS of about $100 \mathrm{~nm}$ needed for the representation of the redness of the Sun at Sunset (see next sections for confirmations).

4) Evidence that the IRS of the blue light also exists for all other frequencies, although with different intensities depending on frequencies according to known laws, thus providing additional preliminary evidence of the IRS origin of the shift of the entire spectrum of Sunlight at Sunset and Sunrise

\footnotetext{
${ }^{3}$ As clearly stated in the Webster Dictionary, "light scattering" strictly refers to the disorderly dispersal of light in all directions.
} 


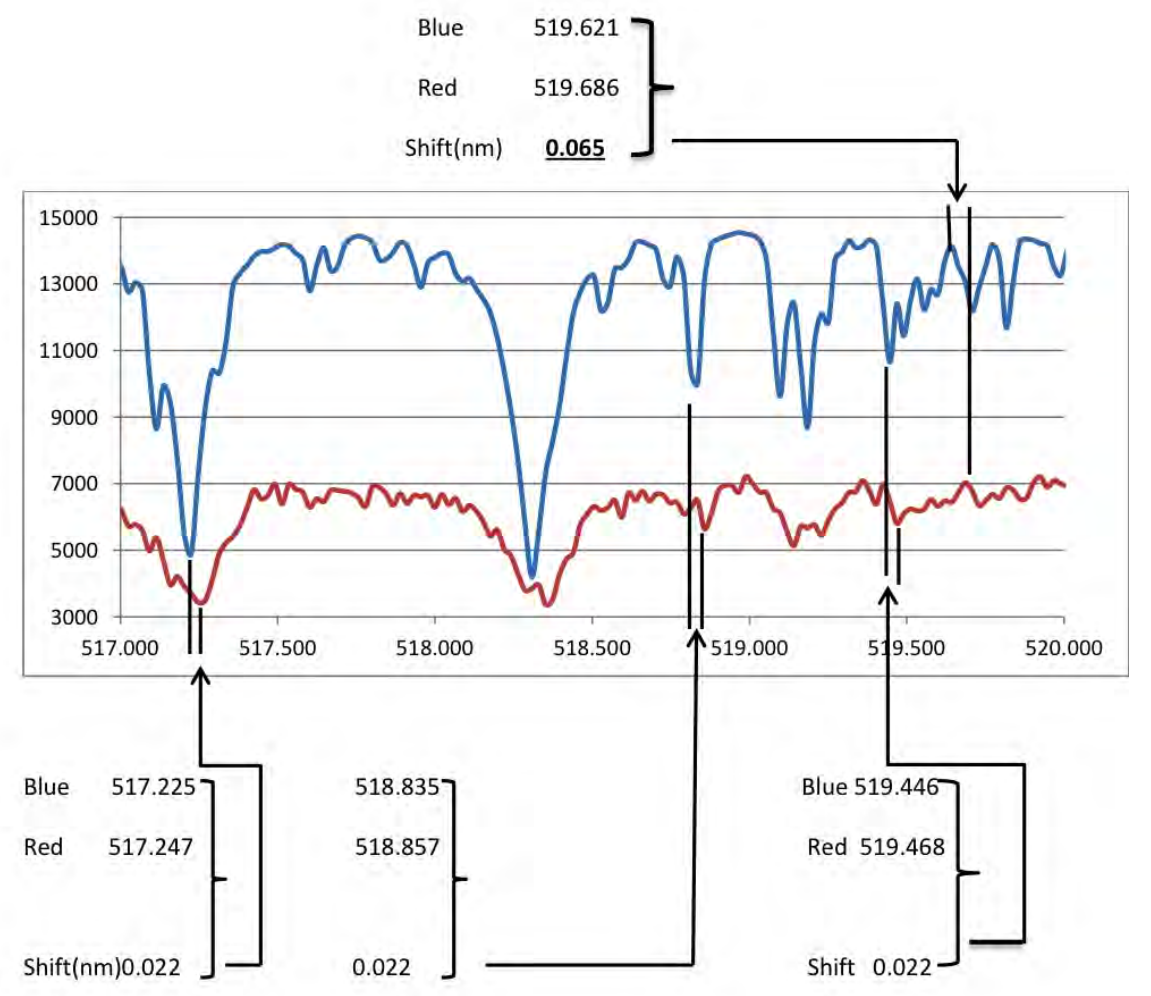

Fig. 14. Spectrograms of representative wavelengths of the Sun at the Zenith (left) and at about $60^{\circ}$ elevation on the horizon (right) establishing an IRS of the order of $0.06 \mathrm{~nm}$. The significance of these measurements is that, at said elevation, no redshift of the Sun is visible to the naked eye. With the decrease of the elevation, the IRS of individual wavelengths increases to such a value that the originally detected spectral lines acquire the characteristics of subsequent spectral lines with no apparent IRS, thus requiring the analysis of the entire sunlight.

as merely due to loss of energy by light to our atmosphere that, after all, experiences an increase of temperature when exposed to Sunlight.

5) The confirmation of Zwicky's hypothesis [15] on the cosmological redshift of far away galaxies as being due to loss of energy by light to intergalactic gaseous media, although according to a mechanism different than scattering as per the original proposal [15]. Consequently, Santilli [9] argued that the IRS of the blue laser light without any relative motion between the source, the medium and the analyzer constitutes experimental evidence on the absence of the expansion of the universe [16], the absence of the acceleration of the expansion [17], the absence of the the big bang [18], and the absence of the expansion of space itself [19] (see Section 10 for details).

6) A numerically exact and time invariant (due to the universal LPS isosymmetry) representation of Hubble's law [20] on cosmological redshift as being mainly due to Santilli IRS, which representation merely requires the use of a specific realization of the average cosmological index of refraction $n_{4}$ for intergalactic gases.

7) The lack of existence of dark matter [21], not only because it would imply a contraction of galaxies from Newton's and Einstein's gravitation, but also due to the lack of a numerical representation of the anomalous redshift of peripheral galactic stars for uniformly distributed dark matter (the 

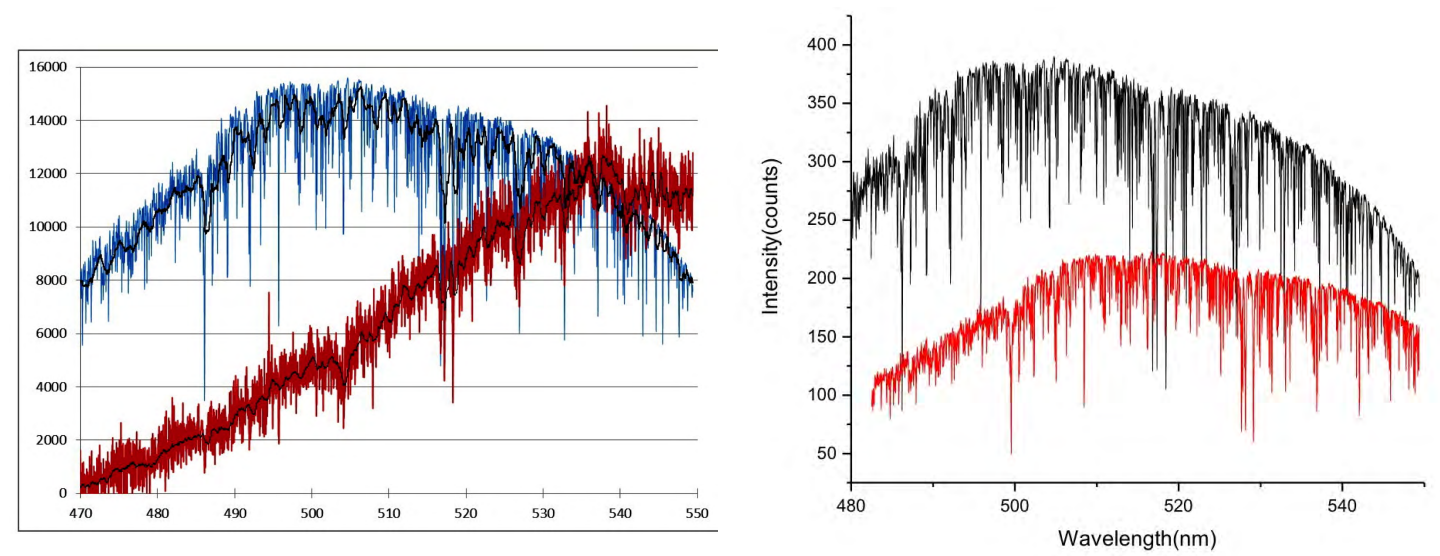

Fig. 15. Illustrations from Ref. [14] of the need for the redshift of the Sun at the horizon to represent the entire spectrum of Sunlight, rather than individual spectral lines or a spectral segment. The top view shows the scan of Sunlight at the Zenith and at Sunset, both restricted from 480 to $580 \mathrm{~nm}$ showing the expected apparent absence of redshift because each line has moved toward the red, thus assuming the same configuration of the corresponding line at the Zenith, the sole visible difference being the intensity that evidently decreases for the Sun at Sunset. The bottom view shows in the top the actual Sunlight at the Zenith from 480 to $580 \mathrm{~nm}$ and the same light on the bottom manually shifted $43 \mathrm{~nm}$, resulting in the same apparent absence of redshift in violation of its very construction.

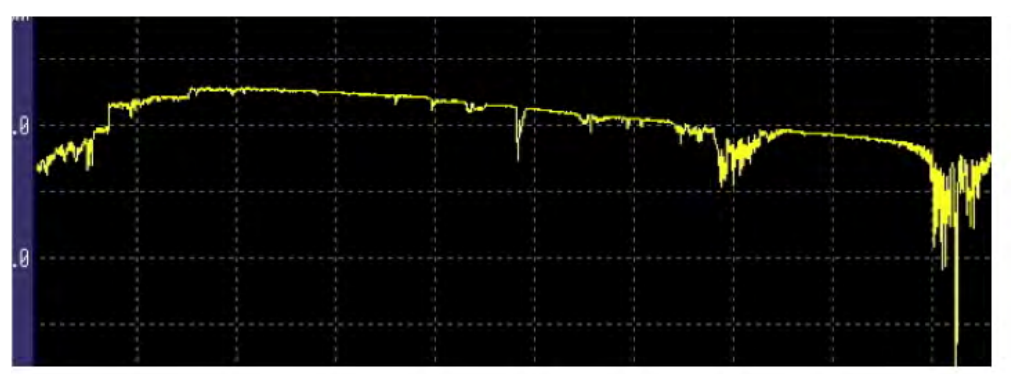

Fig. 16. Chromatogram of the entire spectrum of Sunlight at the Zenith (top) and the main part of the resulting spectrum over background at the horizon (bottom) obtained via the Yokogawa wavelength analyzer model AQ6373 with a range from 400 to $1100 \mathrm{~nm}$ by Santilli, West, and Amato [14] on March 16, 2012.

lack of existence of dark matter is also shown by various other authors, e.g., Ref. [22] and quoted literature).

8) The IRS representation of the anomalous redshifts of peripheral stars for a given galaxy, which is known to vary for galaxies having different configurations, resulting in direct information on the density, inhomogeneity and anisotropy of the gaseous medium within the considered galaxy.

9) The lack of existence of dark energy [23] as a mere consequence of the lack of existence of the expansion of the universe, of its acceleration and all that. 


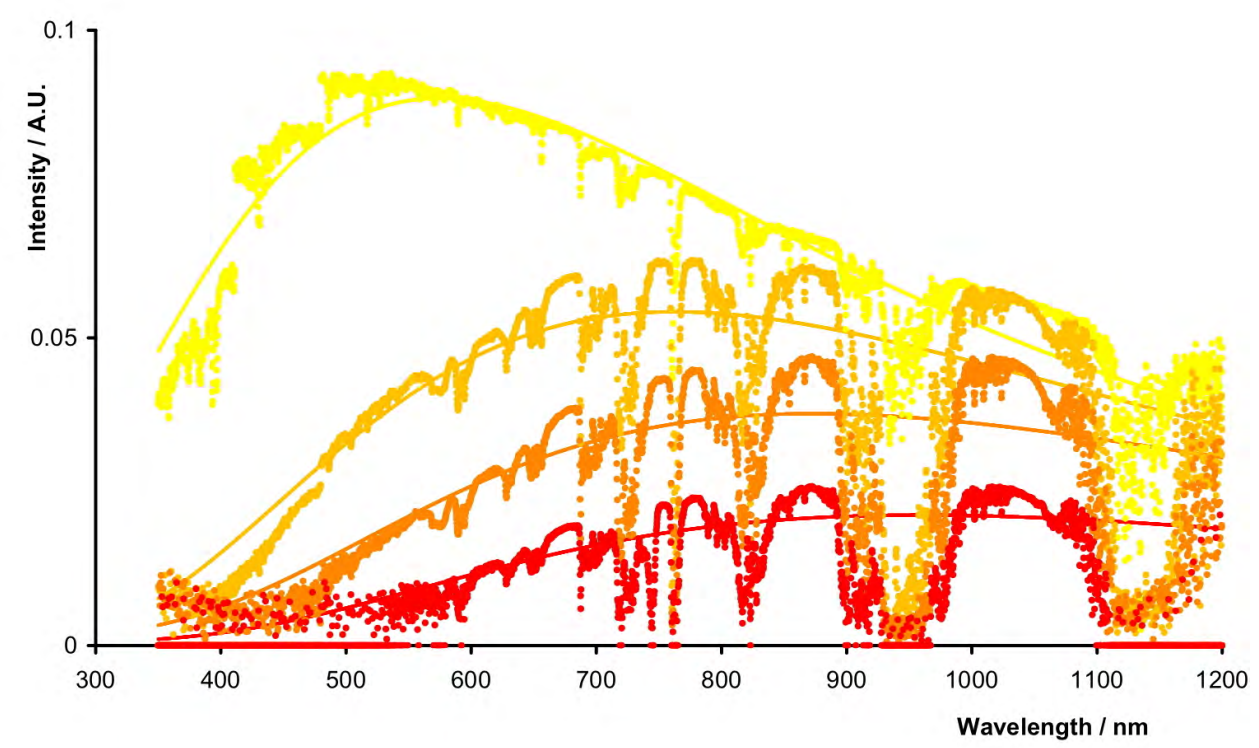

Fig. 17. Chromatographic analysis of the entire spectrum of direct Sunlight obtained in March, 2012, with the Yokogawa analyzer AQ6373 from the Zenith to the horizon presented in Fig. 16 confirming the IRS as the primary origin of the redness of the Sun at Sunset due to a frequency shift of about $100 \mathrm{~nm}$ of the blue light into the red light and also a shift of about $100 \mathrm{~nm}$ of the red light into an infrared band that did not exist for sunlight at the Zenith.

In summary, Santilli's primary motivation for the conduction of decades of the mathematical, theoretical and experimental research herein reported is to avoid a return of cosmology to the Middle Ages with Earth at the center of the universe, which is a necessary consequence of Hubble's law according to which the cosmological redshift as well as its increase are the same for all galaxies having the same distance from Earth in all directions in space. ${ }^{4}$

To close this section with a historical note, Zwicky assumed in his 1929 paper [15] that light would lose energy due to scattering with intergalactic gases. Zwicky's hypothesis was dismissed on various grounds, such as the fact that scattering would imply the inability for galactic lights to reach us, the impossibility of applying scattering for direct galactic light, and other objections that, as we shall see in the next sections, are quite sound indeed (see Section 9 for details).

An implication of Santilli's decades of mathematical, theoretical and experimental research on the IRS is that of having established a more plausible mechanism for light losing energy during its travel within gases, resulting via experiments conducted on Earth, a mechanism today known as the Zwicky-Santilli effect, with consequential return of cosmology to Galileo's teaching.

The reader is expected to be aware that the IRS interpretation of the cosmological redshift will imply a revision of the virtual entirety of the astrophysical and cosmological models accumulated during the past century, beginning with a revision of the currently believed distances of galaxies, a revision of their luminosity at the origin, and other data.

\footnotetext{
${ }^{4}$ The far reaching conjecture that space itself is expanding was voiced precisely to avoid Earth being at the center of the universe. Such an intent would have been achieved in the event the universe was uniformly expanding. By contrast, the acceleration of the expansion implies Earth, again, at the center of the universe despite the expansion of space itself, because said acceleration is the same for all galaxies having the same distance from Earth in all direction in space.
} 


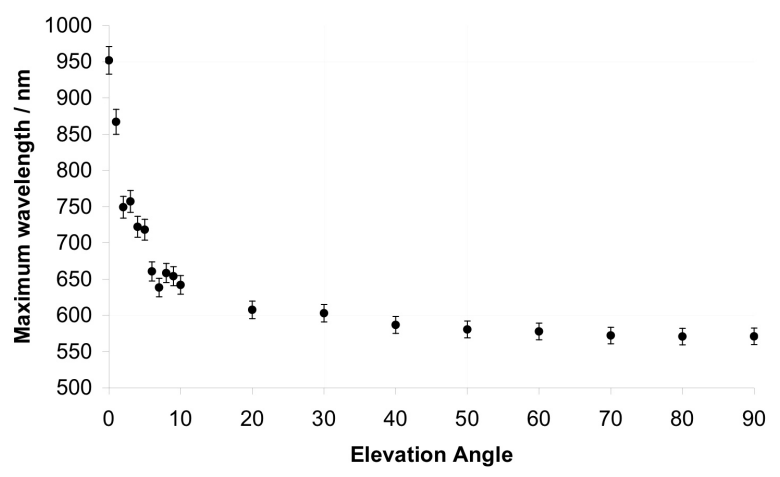

Fig. 18. Chromatographic analysis of all scans of direct Sunlight from the Zenith to the equator reported in Fig. 16 confirming the existence of an IRS of about $200 \mathrm{~nm}$ [14].

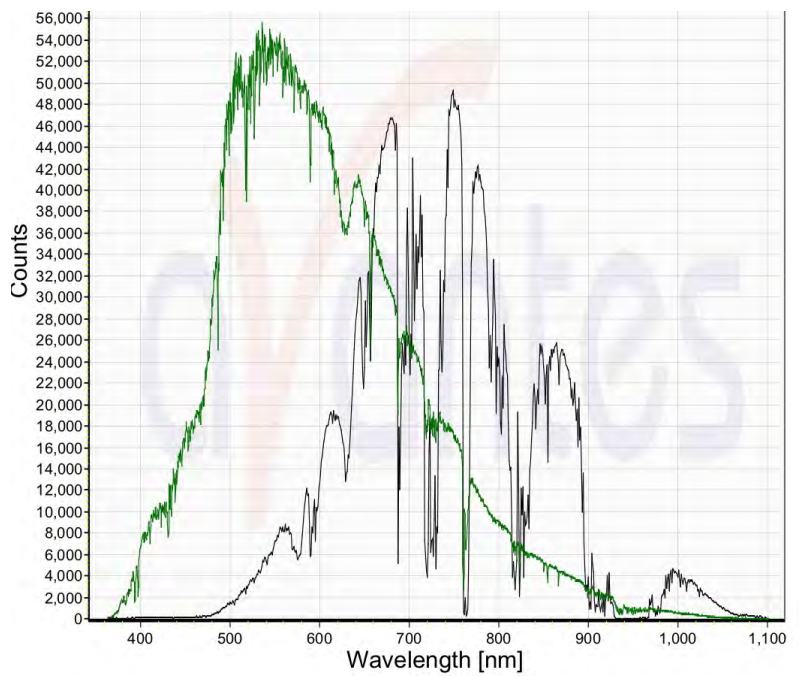

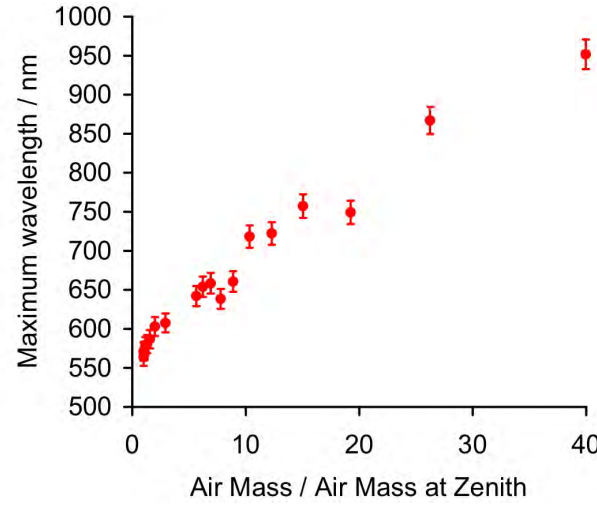

Fig. 19. Yet another chromatographic analysis of all scans obtained via the Yokogawa analyzer AQ6373 of direct visible Sunlight from the Zenith to the horizon as reported in Fig. 16 confirming an IRS of about $200 \mathrm{~nm}$ (see Ref. [14] for details).

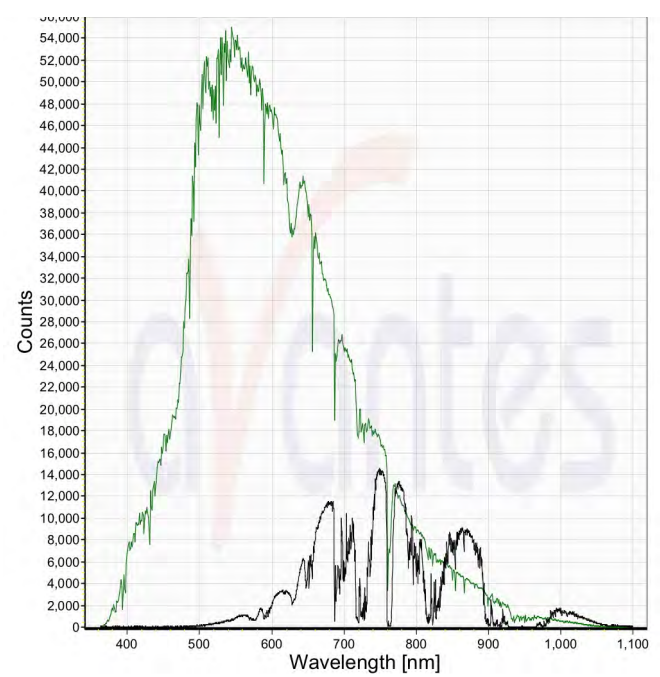

Fig. 20. Representative chromatographs of Sunlight from the Zenith to the horizon obtained on April 22, 2012, via the use of the Avantes wavelength analyzer model AvaSpec 3648 capable of detecting Sunlight frequencies from 400 to $1100 \mathrm{~nm}$ clearly showing the shift toward the red of the entire spectrum of Sunlight, including the expected lack of frequency shift of absorption lines, thus confirming the preceding measurements via the Yokogawa wavelength analyzer model AQ6373.

\section{Second experimental confirmation of Santilli IsoRedShift}

Following the appearance of measurements [9], West and Amato [12] provided systematic experimental confirmations of the existence of Santilli IRS (see the representative scan of Fig. 6), as well as the first experimental evidence on the existence of the IBS (Figs 7. 8), the NIS (Fig. 9) and of the dependence of the IsoShift on the temperature (Fig. 10). via the same IsoShift Testing Station used for measurements [9] (see also Ref. [13]).

West and Amato also provided experimental confirmation of the absence of an appreciable frequency shift for the reflection of the blue laser light on a polished metal mirror under increased temperature, all 


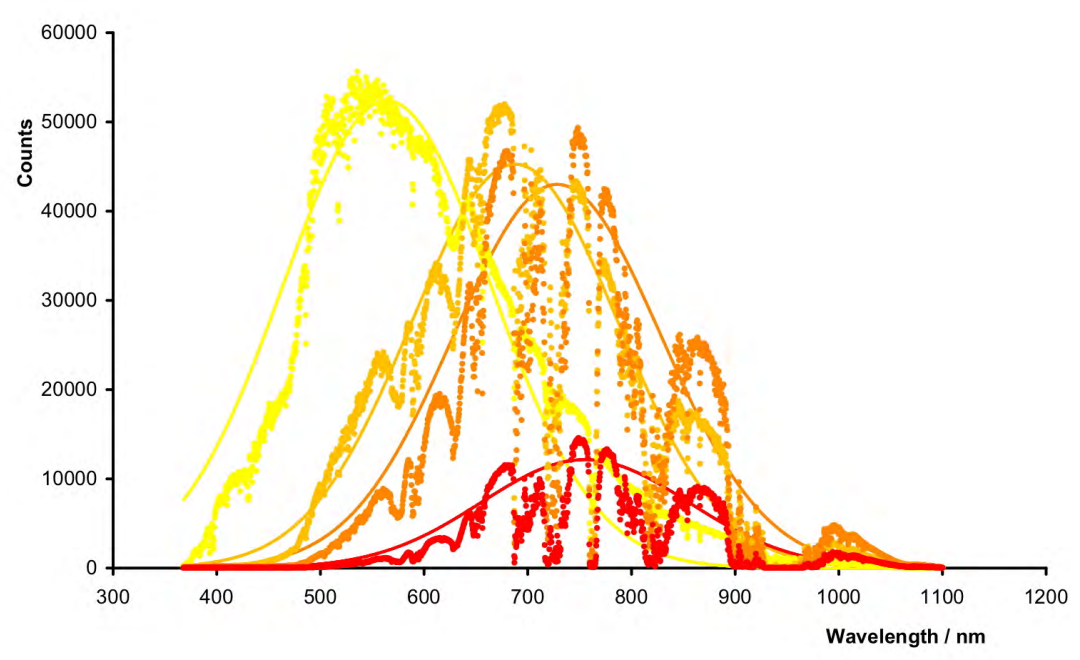

Fig. 21. Confirmation of the elaboration of scans as in Fig. 17, this time obtained via the measurements of Fig. 20 [14].

the way to near the melting value (Fig. 11). The latter measurements were done to confirm that elastic scattering produces no frequency shift, as assumed in Ref. [9]. ${ }^{5}$

These measurements were then used in Ref. [12] to confirm Santilli's 1991 [7b] representation of energy transfer between electromagnetic waves and gaseous media via the Minkowski-Santilli isogeometry and its universal LPS isosymmetry.

As it was the case for Ref. [9], the measurements presented in Ref. [12] are intended to confirm the existence of Santilli's IRS, IBS, and NIS. No statistics and related errors could be provided due to the impossibility of maintaining the same conditions in the IsoShift Testing Station (Fig. 3), due to uncontrollable variations in temperature, leaks of air at pressure in the pipe, and other factors.

Note finally that the measurements of Ref. [12] were strictly referred to direct blue laser light by avoiding significant contributions from the portion of said light which is scattered in all directions inside the tube via the use of collimators, slits and other means (see footnote ${ }^{3}$ ). This leaves as the sole plausible occurrence the loss (or acquisition) of energy by light to (from) the air inside the tube, and the underlying local mutation of the Minkowski spacetime into the covering Minkowski-Santilli isospacetime.

\section{Third experimental confirmation of Santilli IsoRedShift}

Santilli constructed the IsoShift Testing Station and conducted measurements [9] of the IRS for blue laser light in the pipe with air at pressure, specifically, in preparation of measurements on Sunlight from the Zenith to the horizon.

For this purpose, Santilli et al. [14] first established the existence of small IRS values of individual spectral lines of the Sun in the transition from the Zenith to $60^{\circ}$ elevation over the horizon (Fig. 14). However, with the decrease of the elevation, the IRS progressively increases to such values that the

\footnotetext{
${ }^{5}$ An instructive lecture on confirmatory measurements [12] has been provided by G. West and G. Amato in the World Lecture Series available for free viewing or downloading from the link http://www.world-lecture-series.org/san-marino-2011 following its delivery at the 2011 San Marino Workshop on Astrophysics and Cosmology for Matter and Antimatter.
} 


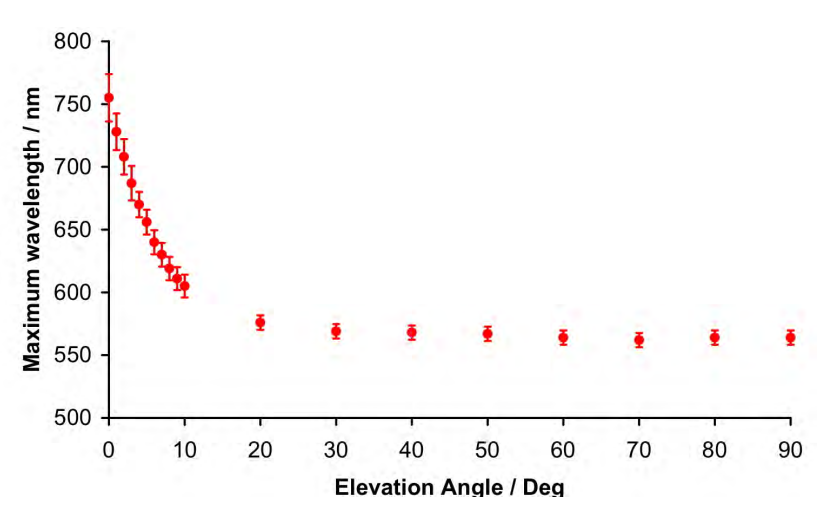

Fig. 22. Confirmation of the elaboration of scans as in Fig. 18, this time obtained via the measurements of Fig. 20 [14].

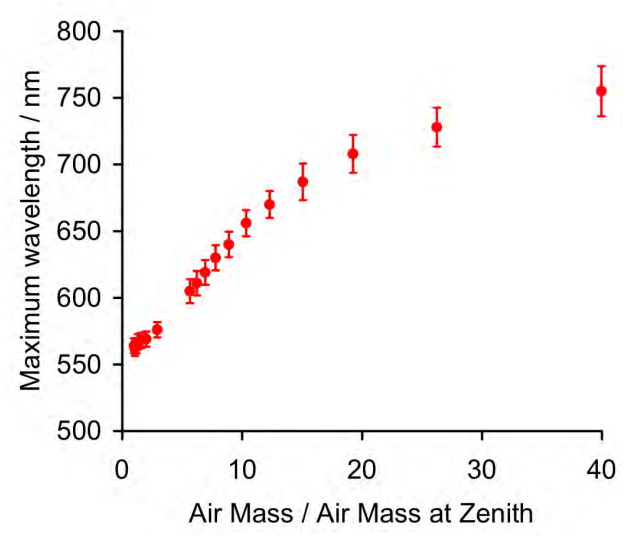

Fig. 23. Confirmation of the elaboration of scans as in Fig. 19, this time obtained via the measurements of Fig. 20 [14].

original spectral lines acquires the characteristics of subsequent spectral lines resulting in no apparent IRS.

To clarify this aspect, Ref. [14] reports spectrographic measurements of Sunlight from the Zenith to the horizon via an Avantes, wavelength analyzer model Avaspec-3648-USB2-FCPC capable of detecting frequencies from 450 to $570 \mathrm{~nm}$ (from green to red). Representative scans at the Zenith and at the horizon are presented in the top view of Fig. 15 showing no apparent IRS, except for the evident decrease of intensity for Sunlight at the horizon.

To show the fallacy of the assumption of no IRS, Ref. [14] reports an artificially made composition (see the lower view of Fig. 15) comprising in the top the actual Sunlight at the Zenith and at the bottom the same light but manually shifted of $43 \mathrm{~nm}$ toward the red, again with no apparent IRS, contrary to its very construction.

These preliminary measurements established the need to study the entire spectrum of visible Sunlight in the transition from the Zenith to the horizon. For these reasons, Santilli et al. [loc. cit.] secured a Yokogawa wavelength analyzer model AQ6373 with the range from 400 to $1100 \mathrm{~nm}$ and conducted systematic measurements of direct Sunlight from the Zenith down to the horizon (see Fig. 16 for representative scans).

These measurements established that the entire spectrum of Sunlight experiences a shift toward the red for about $100 \mathrm{~nm}$, plus there is the appearance of about $100 \mathrm{~nm}$ in the infrared at the horizon that was not present at the Zenith. Figures 17, 18, and 19 present additional confirmatory analyses of the scans reported in Fig. 16 (see Ref. [14] for details).

Additionally, Santilli et al. [14] conducted confirmatory measurements of the scans obtained via an Avantes wavelength analyzer model AvaSpec 3648 capable of detecting Sunlight frequencies from 400 to $1100 \mathrm{~nm}$. The measurements were conducted in the same location (St. Petersburg Beach, Florida West Coast), also from the Zenith to the horizon, and at the same sequence of elevation over the horizon as done for the Yokogawa analyzer. Analysis of the new raw data was also done with the same methods as those used for the preceding measurements.

Representative results of the latter measurements are reported in Figs 20, 21, 22, and 23, with all raw data and other information available for free pdf download from the references of paper [14]. Again, the 

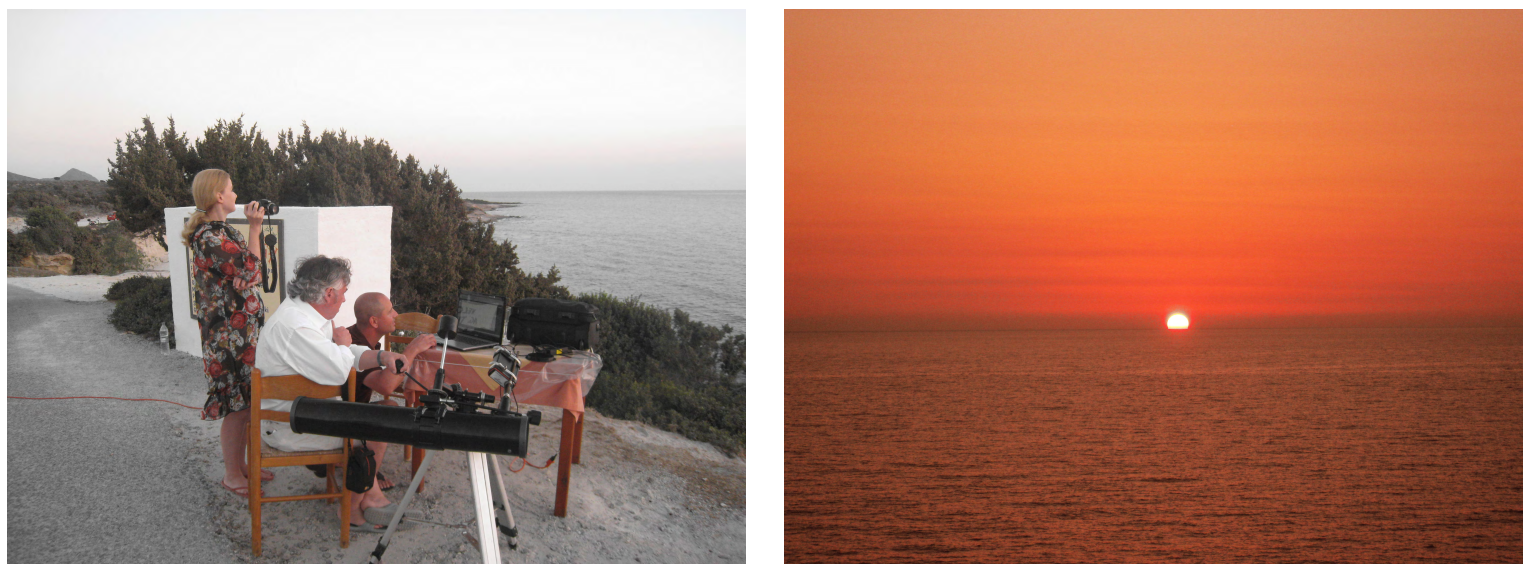

Fig. 24. Pictures of the measurements at the island of Kos, Greece, on September 20, 2012, showing the team preparing the instrument, the analyzer and the computer for recording the scans (top view) and the Sun at Sunset (bottom view).

scans via the Avantes analyzer show quite clearly the shift of the entire spectrum of the Sun toward the red, thus providing a clear confirmation of its IRS origin.

Note that all scans show lack of frequency shift of the absorption lines as expected, because they are set by the chemistry of the atmosphere. Therefore, the only possibility of achieving frequency shift via absorption lines would be to change the entire Earth's atmosphere.

It should also be indicated that the telescope of Ref. [14] was selected to have a small visual angle for the specific scope of restricting the measurements to direct Sunlight, with ignorable contributions from the component of Sunlight scattered throughout the atmosphere.

Ref. [14] also provided a quantitative and time invariant representation of the IRS at Sunset via Eq. (5) with the minus sign for the Doppler shift (because the observer moves away from the Sun at Sunset) and with the minus sign for the Santilli IRS (so as to achieve an overall redshift).

For this purpose, Ref. [14] first noted that the motion of the observer away from the Sun at Sunset (ss) with a tangent velocity at the equator and at sea level of the order of $30 \mathrm{~km} / \mathrm{s}$, thus yielding the conventional Doppler's shift at Sunset

$$
\frac{v}{c} \approx 1.43 \times 10^{-6}
$$

thus being dramatically insufficient alone to represent the redness of the Sun at Sunset.

Next, Ref. [14] used Santilli's isotopic law Eq. (16) and achieved the desired quantitatoive and time invariant representation of vthe redness of the Sun at Sunset, here referred to the IRS of the blue into the red light

$$
\Delta \lambda=\lambda_{\text {irs }}-\lambda_{\text {blue }}=\frac{v}{c}\left(1+K_{s s} d+\ldots\right) \lambda_{\text {irs }}=K_{s s} d \frac{v}{c} \lambda_{\text {irs }} .
$$

where the subscript ss stands for Sunset, the isolated term representing the contribution of the Doppler shift is ignored because it is much smaller than the IRS, and the expression is referred to the wave front of Figs 18 and 22.

By using the various numerical values of the event, the full Doppler-Santilli isoshift becomes [14]

$$
\Delta \lambda=K_{s s} d \frac{v}{c} \lambda_{i r s}=K_{s s} 70001.4310^{-6} 650 \mathrm{~nm}=100 \mathrm{~nm} .
$$

with numerical value for the constant $K_{s s} \approx 15 \mathrm{~km}^{-1}$ band corresponding half value for the $100 \mathrm{~nm}$ shift of the blue to the red. 


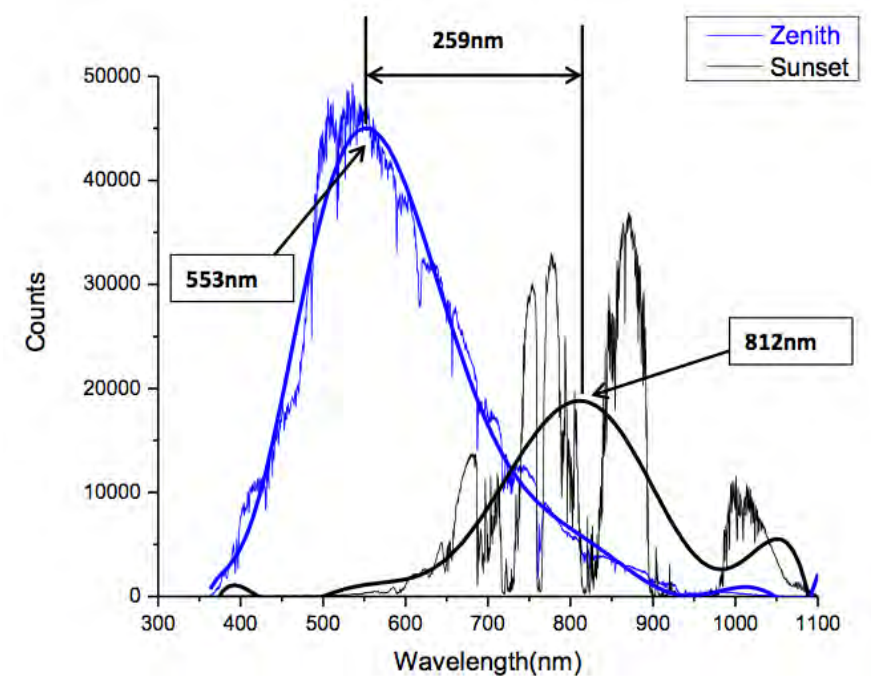

Fig. 25. Representative chromatographs of Sunlight from the Zenith to the horizon obtained on September 20, 2012, at the island of Kos, Greece, via the use of the Avantes wavelength analyzer model AvaSpec-ULS2048 providing a clear confirmation of the chromatographic analyses conducted in Florida as per Figs 16 and 20. Raw data for independent verifications are available to interested colleagues for free download from the link of Ref. [24].
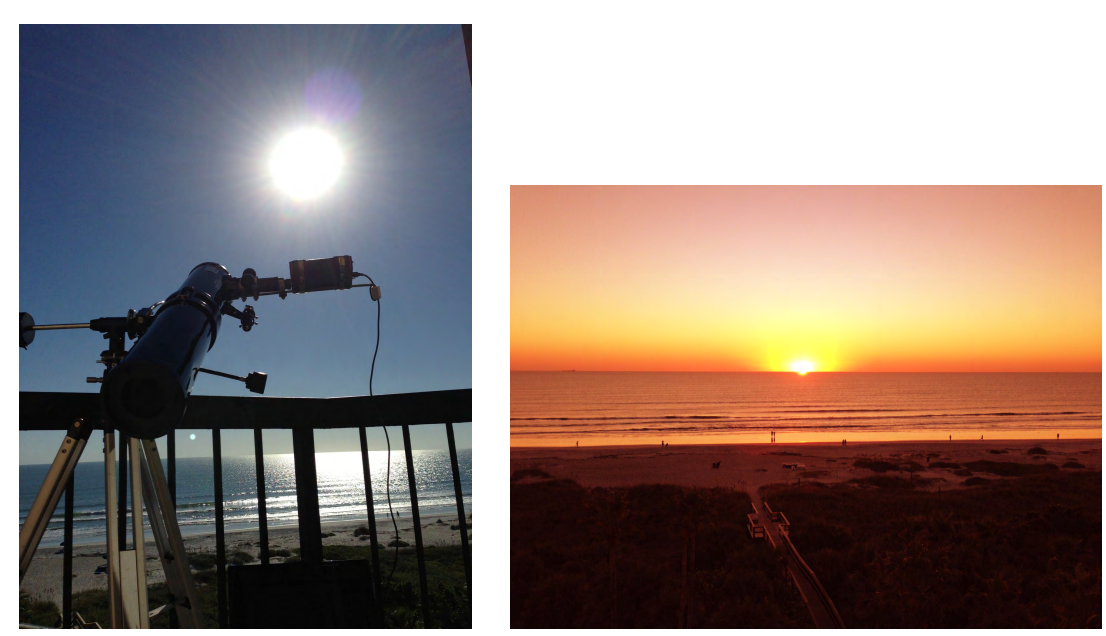

Fig. 26. Pictures of the measurements at Cocoa beach, Florida, on October 20, 2012, showing the experimental set up (left view) and the Sun at Sunrise (right view).

In summary, Ref. [14] established that Santilli's IRS formalism provides a numerical representation of large frequency shifts of Sunlight at Sunset in a way invariant over time thanks to the LPS isosymmetry, namely, in such a way as to predict the same numerical values under the same conditions at different times, thus confirming the mutation of the spacetime of the vacuum caused by matter.

As we shall see in more details in Section 9, the hypothesis of the absorption of blue light at the horizon for the intent of maintaining the red light is clearly dismissed by the scans reported in Figs 16 and 20, since the frequency band surrounding red at the Zenith completely disappears at the horizon and it is shifted for about $100 \mathrm{~nm}$. 


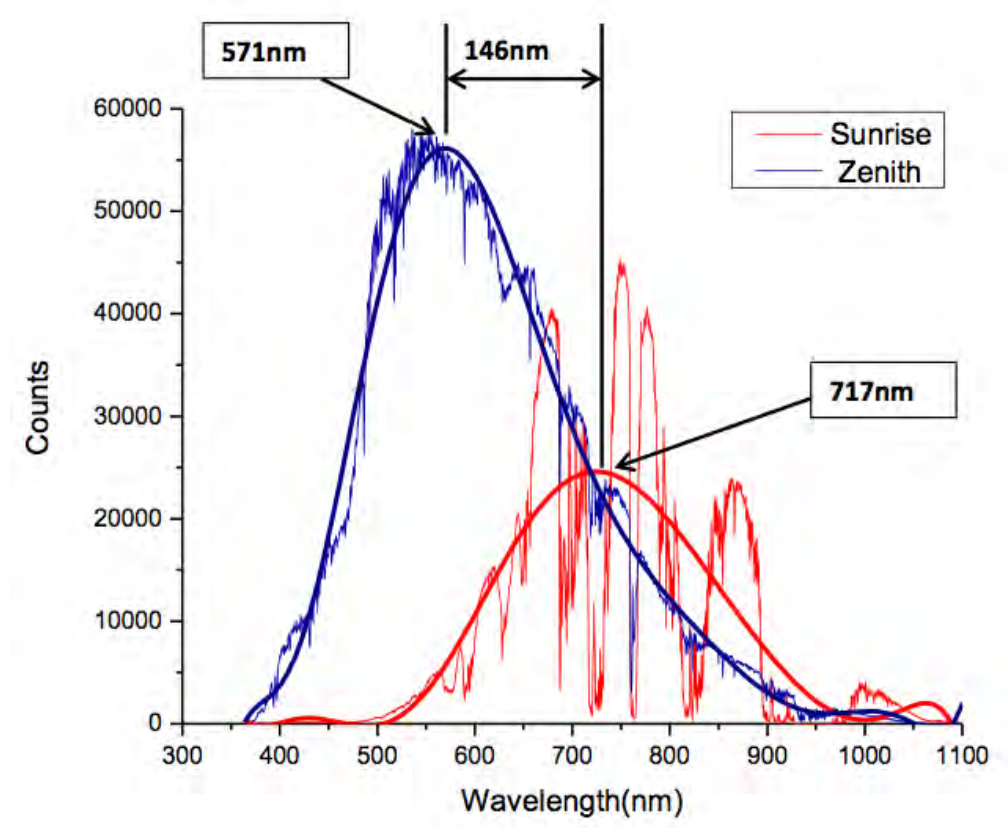

Fig. 27. Representative chromatographs of Sunlight from Sunrise to the Zenith obtained for the first time by the authors on October 20, 2012, at Cocoa beach in the East Coast of Florida. The scans depict Sunlight at the Zenith in the left and that at lower elevation in the right. However, their order is inverted with respect to the scans for Sunset because the first detection for Sunrise is the redness of the Sun that progressively shifts toward the white for the Zenith. The scans here presented clearly confirm Santilli's IRS of the Sun at the horizon with numerical values essentially the same as those of the preceding scans for the Sunset. Raw data for independent verification are available to interested colleagues for free download from the link of ref. [25].

Scattering evidently occurs for Sunlight at Sunset and it is in fact responsible for the decrease of the intensity of Sunlight according to which part of the original Sunlight in vacuum is scattered in all directions when traveling in our atmosphere, resulting in the visible decrease of intensity. However, scattered light is not considered at all in Ref. [14], since measurements solely refer to direct Sunlight at Sunset, namely, to Sunlight propagating along a straight line for which the very definition of scattering does not apply (see footnote ${ }^{3}$ ).

Needless to say, Ref. [14] does not exclude other interpretations (such as that via Rayleigh and other models) that are complementary and not in conflict with the IRS representation, since the former refer to the description of the intensity shift, while the latter refer to the physical origin of the frequency shift within air derived from the primitive LPS isosymmetry in exactly the same way as the Doppler shift in vacuum is derived from the primitive LP symmetry.

In fact, Santilli's IRS formalism provides a representation via the iso-Minkowskian geometry of the redness of Sunlight at Sunset by predicting in a unique and invariant way the physical origin of said redness as being due to loss of energy by light to our atmosphere. Along fully similar lines, the Minkowskian geometry provides a unique and invariant representation of the frequency shift in vacuum as being due to relative motion between the source and the observer.

Recall that at Sunset we have the IRS, rather than the IBS, because light travels in air which is very cold for the greatest part of the trajectory. Rather intriguingly, Santilli [7b] predicts that planets of other solar systems can indeed experience a blue shift for starlight at the horizon in the event its atmosphere is sufficiently hot, namely, the planet is sufficiently close to the star. 


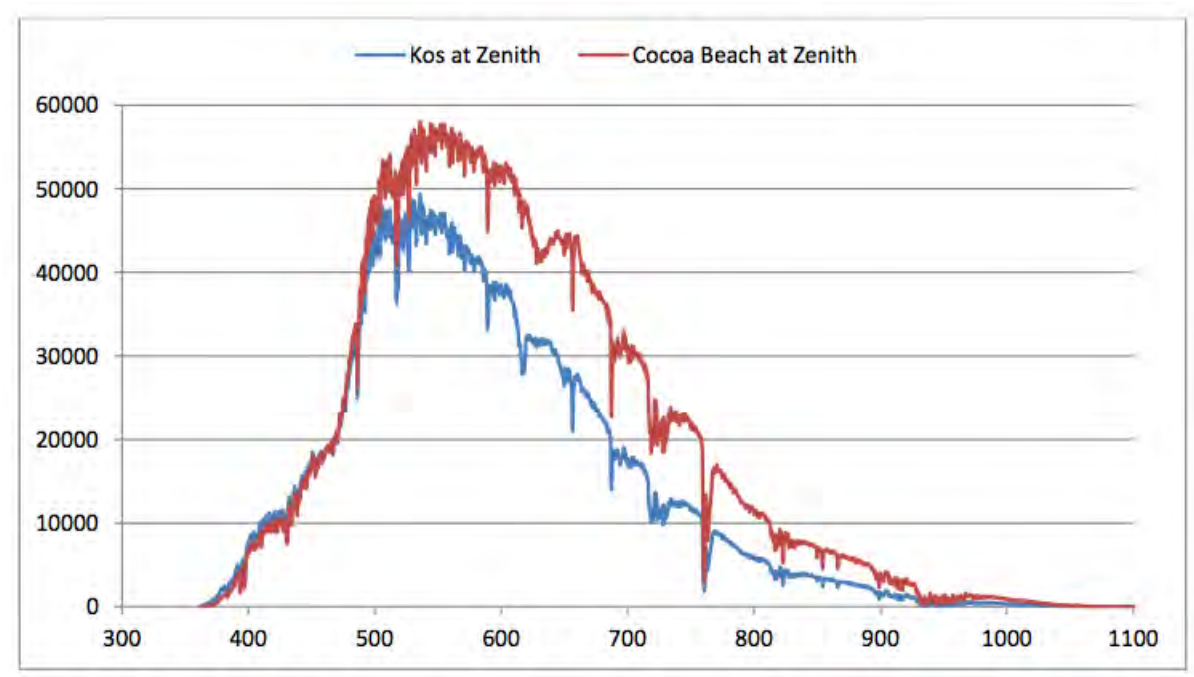

Fig. 28. The Sun at the Zenith in two different locations, at Kos, Greece and at Cocoa Beach Florida. The small difference in the intensity is due to the manual orientation of the telescope and it is fully within the range of accurate measurement of frequencies by the Avantes analyzer. Even assuming that such a variation of intensity may affect frequencies, the related numerical value would be ignorable over the large IRS from the Zenith to the horizon.

\section{Fourth experimental confirmation of Santilli IsoRedShift}

The fourth verification of Santilli's IRS origin of the redness of the Sun at the horizon was conducted by the authors on September 20, 2012, on the southwest beach side of the island of Kos in the Kefalos town, Greece, for the first time in the transition of the Sun from the Zenith to the horizon (Sunset).

We began recording Sunlight at the Zenith and repeated the recording thereafter for every $10^{\circ}$ decrease of the elevation over the horizon. At $10^{\circ}$ elevation we conducted scans at every degree of decrease of the elevation or every four minutes. The first scan was recorded at 1:14 pm and the final scan was recorded at 7:11 pm. Representative scans are reported in Fig. 24, while all raw data is made available for independent analyses for free download from the link of Ref. [24].

All scans were conducted with an Avantes wavelength analyzer model AvaSpec 3648 capable of detecting Sunlight frequencies from 400 to $1100 \mathrm{~nm}$ and with a selectable integration time range between $0.01 \mathrm{~ms}$ and $600,000 \mathrm{~ms}$. We selected the integration time of $20 \mathrm{~ms}$ because it allowed to consistently capture Sunlight from the lower intensity at the horizon all the way to the Zenith without over-saturating the analyzer. ${ }^{6}$

All conditions were essentially the same as those of Ref. [14]. For instance, the authors took particular care in selecting a telescope with an aperture to capture direct Sunlight with insignificant contributions from Sunlight scattered by the atmosphere surrounding the Sun at all elevations.

Comparison of our results with the preceding ones [14] establishes the achievement of the same numerical values of Santilli's IRS, namely, about $100 \mathrm{~nm}$ frequency shift of the blue to the red, plus the appearance of about $100 \mathrm{~nm}$ in the infrared at the horizon that was not detectable at the Zenith.

\footnotetext{
${ }^{6}$ For instance, the integration time of $10,000 \mathrm{~ms}$ would certainly be able to capture the lower intensity light at zero degrees elevation, but the analyzer would have been progressively saturated at bigger elevations. Similarly, the integration time of $0.01 \mathrm{~ms}$ would be indeed capable to capture Sunlight at high elevation, but would have been unable to provide any meaningful recording at very low elevations due to insufficient sensitivity.
} 


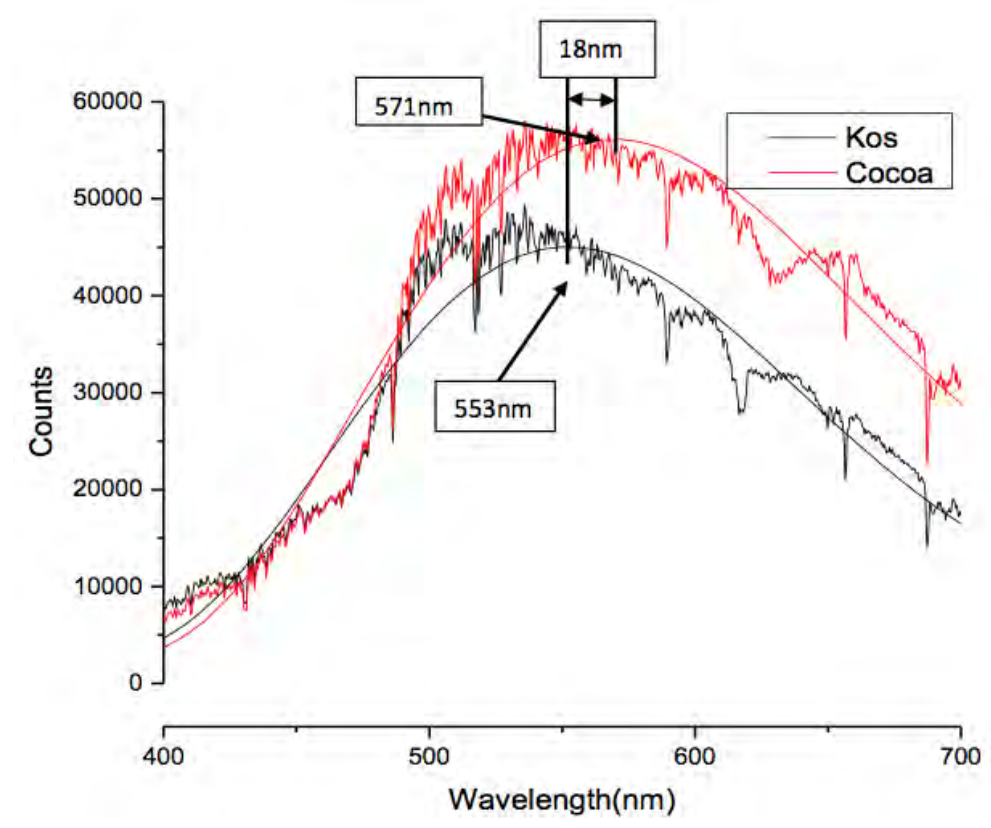

Fig. 29. A close up analysis of Sun spectra at the peaks from Fig. 29. The polynomial fit shows a $18 \mathrm{~nm}$ shift in said peaks that are significant as they may provide information on the expected dependence of the IRS on local variations of our atmosphere, variations due to different latitudes and longitudes, possible differences in the actual angle of detecting spectra at the Zenith, and other reasons.

Consequently, our measurements on the island of Kos, Greece, provide a fourth clear experimental confirmation that the redness of the Sun at Sunset is dominated by Santilli's IRS, except for a small contribution of the order of $10^{-6}$ for the conventional Doppler's effect due to the motion of the observer away from the Sun at Sunset caused by Earth's rotation (see the preceding section for details).

One of the most important implications of our measurements is to provide an additional experimental confirmation of the mutation of the Minkowski spacetime for the vacuum into the covering MinkowskiSantilli isospacetime for our atmosphere, with consequential applicability of the covering LPS isosymmetry and related isorelativity.

\section{Fifth experimental confirmation of Santilli IsoRedShift}

The fifth verification of Santilli's IRS origin of the redness of the Sun at the horizon was conducted by the authors on October 20, 2012, in Cocoa Beach, Florida on the East Coast, for the first time in the transition of the Sun from the horizon (Sunrise) to the Zenith.

All equipment used in this test was the same as that of the preceding measurements on the island of Kos (Section 7), including the same telescope, analyzer, setting and procedures, except that the latter ones were reversed, e.g., following Sunrise we scanned the Sun for every degree of increasing elevation up to $10^{\circ}$ and, thereafter we conducted scans for every additional $10^{\circ}$ all the way to the Zenith.

Santilli's IRS was inverted too, in the sense that, for the measurements from the Zenith to Sunset we have the shift of blue light all the way to red, while for the measurements of Sunrise we have the inverse shift of the original red light all the way to the blue. Note that the latter inverted recording does not imply 


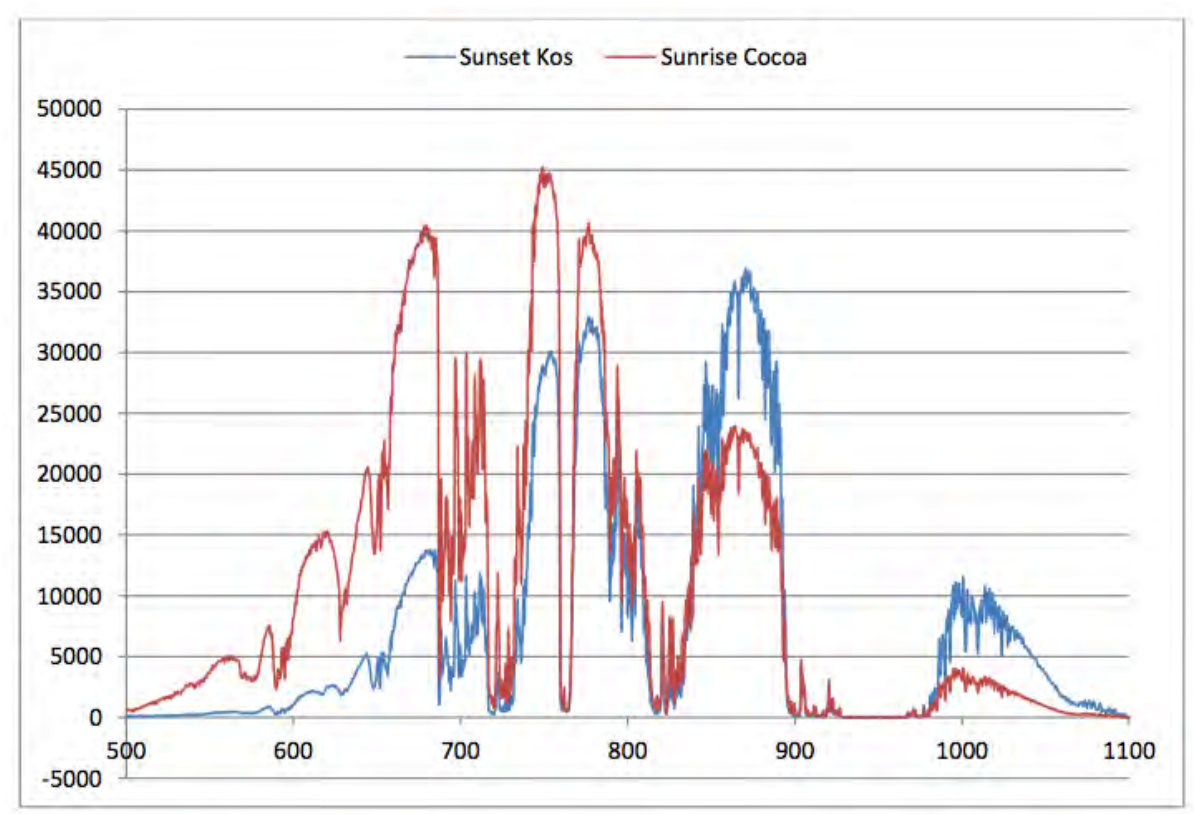

Fig. 30. Sunset at Kos Island vs. Sunrise at Cocoa beach. There appears to be matching wavelengths of peaks and valleys for Sunrise and Sunset regardless of the location of the country, and regardless of the motion/rotation of the Earth, thus confirming that the conventional Doppler's shift due to opposite rotations at Sunset and Sunrise is ignorable over Santilli's IRS as indicated in Sect. 2 of Ref. [14]. The matching wavelengths confirm the lack of appreciable impact of intensities on frequency detection for the Avantes analyzer provided, of course, that the intensities are sufficient for the detection of frequencies.

the existence of Santilli IBS at Sunrise, since the shift remains to be characterized by a comparison of data at the horizon and at the Zenith.

Comparison of our results for the Sunrise with the preceding ones for the Sunset (Sections 6. 7) establishes the achievement of essentially the same numerical values of Santilli's IRS, namely, about $100 \mathrm{~nm}$ frequency shift of the blue to the red, plus the appearance of about $100 \mathrm{~nm}$ in the infrared at the horizon that was not detectable at the Zenith.

Consequently, our measurements for the Sunrise provide yet another experimental confirmation of the mutation of the Minkowski spacetime for the vacuum into the covering Minkowski-Santilli isospacetime for our atmosphere, with consequential applicability of the covering LPS isosymmetry and related isorelativity.

A quantitative, time invariant representation of the IRS of the Sun at Sunrise (sr) was provided by Santilli et al. in Ref. [14] via general law Eq. (5) specialized to the fact that, due to Earth's rotation, for at Sunrise the observer moves toward the Sun, in which case the sign of the conventional Doppler's shift is plus, while the sign for Santilli IRS is minus.

As a complement of representation Eq. (22) for the Sunset, Santilli et al. [14] obtain the representation for the Sunrise here referred to the the shift from the blue to the red

$$
\Delta \lambda=\lambda_{\text {irs }}-\lambda_{\text {blue }}=-\frac{v}{c}\left(1-K_{s r} d+\ldots\right) \lambda_{\text {irs }}=K_{s r} d \frac{v}{c} \lambda_{\text {irs }}=100 \mathrm{~nm} .
$$

thus providing a numerical value of the constant $K_{s r}$ for Sunrise essentially identical to that for $K_{s s}$ (although slightly smaller due to the opposite Doppler contributions here ignored).

Comparisons of the measurements of IRS done at the Kos Island for Sunset with those done at Cocoa B each for Sunrise (presented in Figs 30 and 31) have confirmed the invariant representation of Solar 


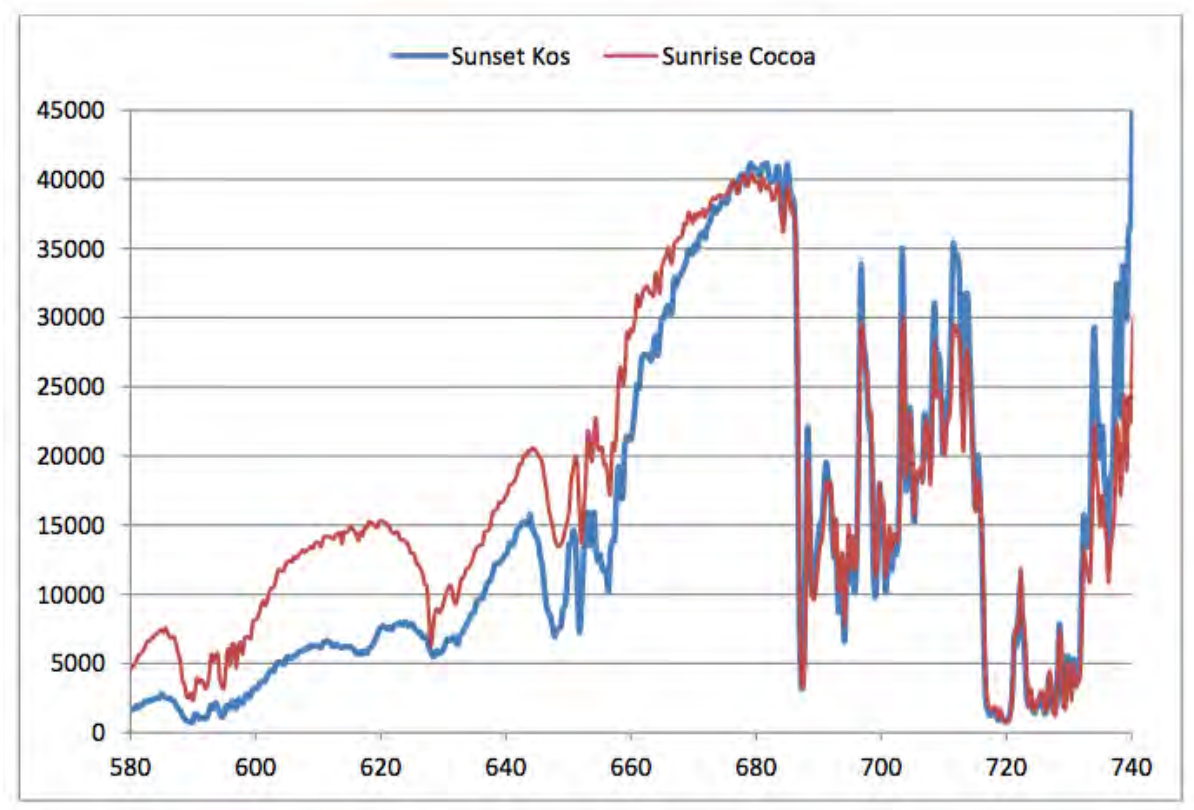

Fig. 31. A close up analysis at the region between 550 and $750 \mathrm{~nm}$ of Fig. 31 with intensities adjusted to the same value confirming a matching wavelengths. The wavelength show symmetry in the conditions as it relates to atmosphere and rotation of Earth between sunrise and sunset.

redness done in Ref. [14], Section 2.8, Eqs (1) to (9), to the effect that the conventional Doppler's shift caused by opposite rotations of Earth during Sunset and Sunrise is excessively small (of the order of $10^{-6} \mathrm{~nm}$ ) compared to the dominance of the IRS (for about $100 \mathrm{~nm}$ ) and, consequently, can be ignored in first approximation.

\section{Expected absence of the universe expansion and all that}

\subsection{Historical notes}

Santilli has dedicated decades of mathematical, theoretical and experimental research to honor the view by E. Hubble, F. Zwicky, L. de Broglie, and other famous physicists on the absence of the expansion of the universe because it implies a necessary return to the Middle Ages with Earth at the center of the universe (see the conceptual rendering of Fig. 33).

As it is well known, the uneasiness created by Earth at the center of the universe suggested Zwicky [15] the proposal of the "Tired Light" according to which galactic light loses energy to intergalactic media due to scattering. Unfortunately, the scattering origin of Tired Light was correctly dismissed in the early 20 th century on grounds that it would prohibit direct galactic light from reaching us (from the very definition of "scattering"), and for other reasons. This occurrence left no other alternative in the early 20th century than the interpretation of the cosmological redshift as being due to a Doppler frequency shift, thus implying to the expansion of the universe.

Santilli could not accept any model implying Earth at the center of the universe. Therefore, he supported Zwicky hypothesis that light loses energy to intergalactic media, and dedicated himself to the 


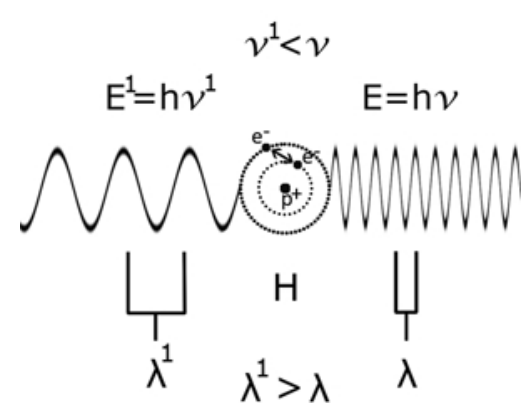

Fig. 32. None of the conventional mechanisms of absorption, scattering, etc. can fully explain the origin of Santilli IRS and IBS. In this picture, we present a schematic rendering of the new mechanism expected for the realization of Santilli's anomalous IsoShifts of galactic light traveling through the intergalactic medium essentially composed of a rarefied Hydrogen gas. The IRS is depicted when inspecting the diagram from right to left. When Hydrogen atoms (assumed to be in their ground state due to low temperature) are hit by light, its transverse structure causes the excitation of the electron with a consequential absorption of energy, thus causing Santilli IRS. Said energy is subsequently expected to be emitted in the form of Cosmic Background Radiation. Santilli IBS is illustrated when inspecting the diagram from left to right. In this case, light is expected to trigger the return to the ground state of atoms in their excited state due to local temperature (such as in astrophysical chromospheres) with a consequential expected release of energy to light and resulting IBS.

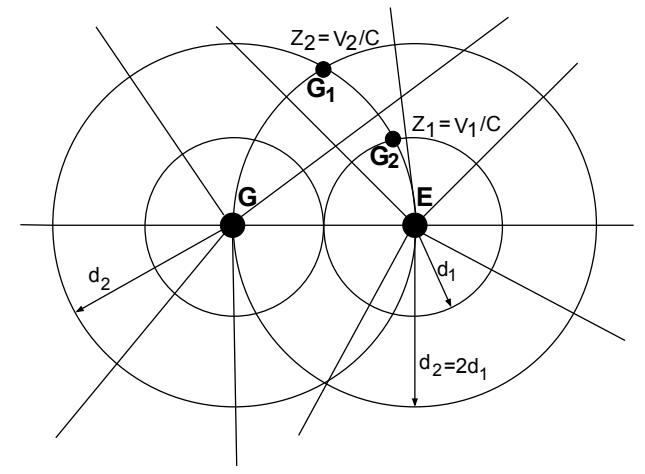

Fig. 33. Santilli's diagram illustrating the rejection by E. Hubble, F. Zwicky, L. de Broglie, and others of the expansion of the universe in view of its acceleration (Fig. 32). Santilli's diagram establishes the inconsistency of the Doppler interpretation of the cosmological redshift because the relative acceleration between galaxies $G_{2}$ and $G_{1}$ solely exist when seen from Earth $E$, and does not exist for the same galaxies $G_{2}$ and $G_{1}$ when seen from another observer such as the galaxy $G$ (see Section 9.2 for details). Therefore, Santilli's diagram confirms Earth at the center of the universe under the Doppler law, by de facto ending one century of related controversies in astrophysics and cosmology.

search for an alternative interpretation (other than that due to scattering as illustrated in Fig. 32) studied in this paper, resulting in what is called now the Zwicky-Santilli effect.

\subsection{The 20th Century Return to the Middle Ages}

To outline the main events, let us recall that Hubble's law (Ref. [20] and Eq. (26) below) establishes the proportionality of the cosmological redshift of galaxies to the distance from Earth, thus implying a necessary acceleration of the expansion with the increase of the distance. Most importantly, as very clear from Law (26) below, the cosmological redshift is the same for all galaxies having the same distance in all directions in space from Earth.

In the event the cosmological redshift had been the same for all distances from Earth (namely, in the event the universe was expanding uniformly), the expansion would be the same for all observers anywhere in the universe and Earth would not be at its center.

By contrast, Earth at the center of the universe becomes necessary from the acceleration of the expansion in all radial directions from Earth. Therefore, Earth at the center of the universe is unavoidable also for the "big bang" as well as for the expansion of space itself (see footnote ${ }^{4}$ ).

In order to better illustrate the view by E. Hubble, F. Zwicky, L. de Broglie and other famous scientists, Santilli presented the diagram of Fig. 33 at the Seminar Course on Hadronic Mathematics, Physics and Chemistry held in the Kos Island in Greece on September 2012. As one can see, Santilli's diagram establishes the inconsistency of the Doppler interpretation of the cosmological redshift because the rel- 
ative acceleration of galaxies is unique to Earth, thus implying Earth at the center of the universe, it is not the same for other observers in the universe.

In fact, under the Doppler interpretation $z=v / c$, Eq. (27), of the Hubble law $z=H d$, Eq. (26), the galaxies $G_{2}$ and $G_{1}$ have the cosmological redshifts $z_{2}=v_{2} / c$ and $z_{1}=v_{1} / c$ with $v_{2}=2 v_{1}$ since $d_{2}=2 d_{1}$, thus implying that the galaxy $G_{2}$ accelerates away from $G_{1}$ when seen from Earth E.

However, when $z_{2}$ and $z_{1}$ are measured from the galaxy $\mathrm{G}$, we have $z_{2}=z_{1}$ since the two galaxies are located at the same distance $d_{2}$ from $\mathrm{G}$, thus establishing that the galaxy $G_{2}$ has no acceleration away from $G_{1}$ when seen from $G_{1}$.

This implies the inconsistency of the Doppler interpretation of Hubble's law that persists under the far reaching conjecture of the expansion of space itself or of any preferred geometry, since the latter must verify Hubble's law, thus having Santilli's diagram in the local tangent plane.

In summary, Santilli's diagram of Fig. 33 ends one century of controversies by disproving the expansion of the universe and related conjectures, but confirms the original conception by Hubble Eq. (26) and, therefore, its interpretation via Santilli's IRS, Eqs (28), (30) and (32).

Note that the interpretation of the cosmological redshift via Santilli's IRS law for galactic light losing energy to cold intergalactic media resolves the inconsistency of Fig. 33, since said law is characterized by distances, rather than by speeds, thus implying $z_{2}=2 z_{1}$ for galaxies $G_{2}$ and $G_{1}$ when seen from Earth, and $z_{2}=z_{1}$ for the same galaxies $G_{2}$ and $G_{1}$ when seen from galaxy $G$ without any inconsistency, precisely in view of the absence of relative speeds and ensuing accelerations.

In any case, the Doppler interpretation of the cosmological redshift has now reached such extremes to cause serious doubts on its plausibility (irrespective from Santilli's objections) due to the need for galaxies at the edge of the known universe to travel faster than the speed of light in vacuum, as a condition to represent the related cosmological redshifts (see also Eq. (27) below and related comments).

These extremely far reaching conditions are evidently eliminated by the interpretation of the cosmological redshift via Santilli IRS law Eq. (28), since in this case the cosmological redshift is proportional to the traveled distance of light within intergalactic media, and not to the velocities violating the very basic axions of special relativity.

Therefore, Santilli's IRS law Eq. (16) (see reformulations (30) and (32) below) consistently represents cosmological redshifts for all observers in the universe, by implying the lack of expansion of the universe and the lack of its acceleration, with the consequential lack of the "big bang", the expansion of space itself and any need for the conjecture of "dark energy".

Note that the far reaching conjecture of the expansion of space itself (see e.g., Ref. [19]) was formulated precisely to avoid Earth at the center of the universe, under the expectation that it would allow the same cosmological redshift for all observers in the universe. However, Santilli's diagram of Fig. 33 establishes that said intended objective was not achieved because the acceleration of the expansion implies in reality different cosmological redshifts for different observers in the universe.

In Ref. [9], Santilli dismissed the "big bang" conjecture on grounds that: 1) It implies Earth at the center of the universe as a necessary condition for compatibility with the universe expansion and its acceleration; 2) By the very definition of explosion, the universe should be without galaxies for a radius around Earth of at least 13 billions light years; and 3) The "debris" of the primordial explosion (the galaxies) should slow down with the increase of the distance from Earth. By contrast, all these features are disproved by astrophysical evidence.

Additionally, Santilli dismissed the interpretation of the Cosmic Background Radiation (CBR) as "evidence" of the "big bang" because said radiation should have been absorbed by galaxies and intergalactic gases billions of years ago precisely in view of its weak character. By contrast, Santilli stressed the need 
for a continuous source of energy for the CBR to exist. This continuous source is indeed provided by the Zwicky-Santilli effect since the energy lost by galactic light during the long travel to Earth is absorbed by hydrogen and other intergalactic gases and, thereafter, re-emitted expectedly in the form of CBR (see Fig. 32).

In Ref. [9], Santilli also dismissed the conjecture that the mysterious "dark matter" surrounds galaxies for numerous reasons, such as: A) The conjecture of "dark matter" does not provide a numerical representation of the anomalous behavior of peripheral galactic stars for which the conjecture was voiced; B) In the event "dark matter" is adapted to have the need distribution for the representation of the dynamics of one peripheral galactic stars, the dynamics of the remaining stars is manifestly disproved by evidence; C) In the event permeated by "dark matter", galaxies should necessarily contract from Newton's universal gravitation, contrary to astrophysical evidence; and for otehr reasons.

Jointly, Santilli provided in Ref. [9] a numerically exact and time invariant representation of the anomalous redshift of peripheral stars of a given galaxy via expressions of type Eq. (16) reviewed below. As a matter of fact, the anomalous redshift of peripheral galactic stars provides important experimental information on innergalactic media that are often visible via telescopes.

Finally, Santilli dismissed in Ref. [9] the additional far reaching conjecture that the universe is composed for about $75 \%$ by a mysterious entity called "dark energy", and dismissed as well the use of Einstein gravitation for the interpretation of the universe expansion, on various grounds, such as:

i) The lack of identification of the immense energy needed for the acceleration of billions of galaxies for billions of years;

ii) The extremely weak gravitational attraction between galaxies due to very large mutual distances. thus preventing a plausible use of Einstein gravitation for intergalactic dynamics;

iii) The impossibility of "adjusting things" via the use of ad hoc coordinates in a Riemannian space because the same coordinates should be valid everywhere, resulting in dramatic disagreements with experimental data; and other inconsistencies.

In view of the above and other problems of consistency and plausibility, it is evident to serious scholars that the 20th century astrophysics and cosmology need a re-inspection.

\subsection{Hubble, Hubble-Doppler and Hubble-Santilli laws}

Let us now review the primary representations of the cosmological redshift following the advent of Santilli IRS and its experimental verifications.

The Hubble law [20] in its original conception and experimental verifications without ad hoc assumptions can be written

$$
z=\frac{\lambda_{o}}{\lambda_{e}}-1=H d
$$

where $\lambda_{o}\left(\lambda_{e}\right)$ is wavelength at the origin (that observed on Earth), $H$ is Hubble's constant (different in value from the constant appearing in other formulations, such as that below), and $d$ is the distance of the considered galaxy from Earth.

The Hubble-Doppler law (see, e.g., ref. [16]) is given by the tacit assumption that redshift Eq. (26) is due to galaxies moving away from Earth, and it is rather universally written in the form

$$
z=\frac{\lambda_{o}}{\lambda_{e}}-1 \approx \pm \frac{v}{c}
$$

where $+(-)$ denotes motion away from (toward) Earth. 
According to the above law, the cosmological redshift is reduced to the relative speed $v$ of galaxy away from Earth. However, it should be stressed that the actually measured quantity is the cosmological redshift of light, since the relative speed $v$ is a pure assumption. Also, as indicated earlier, far away galaxies require $v>c(z>1)$ for the interpretation of their cosmological redshift in violation of the causality law set forth by special relativity.

The first Hubble-Santilli law [3,7,9] is given by law Eq. (14) merely applied to the cosmological redshift

$$
z=\frac{\lambda_{o}}{\lambda_{e}}-1 \approx \pm K_{i g} d
$$

where: $+(-)$ denotes IRS release by light of energy to cold intergalactic media; - denotes the IBS acquisition of energy by light from hot media, as measured for certain stars and quasars; and $K_{i g}$ is the Hubble-Santilli intergalactic constant providing a geometrization of intergalactic media (see below for a second form and Fig. 32).

Note that, in the transition from our atmosphere to intergalactic media, we have a very large reduction of the density of the medium but, jointly, we have a very large increase of travel $d$ of light within said medium, thus resulting essentially similar redshifts for the Sun at Sunset and for galactic light, as illustrated in Fig. 12.

Santilli (Ref. [9] and private communication) also provided a second re-formulation of Hubble's law Eq. (26) based on the average speed of electromagnetic waves within intergalactic media, essentially along Eq. (10),

$$
C_{i g}=\frac{c}{n_{i g}(t, r, v, e, d, \omega, \tau, \ldots)} .
$$

where $n_{i g}$ is the average index of refraction for intergalactic media normalized to the value $n_{i g}=1$ for the vacuum. Therefore, at the galactic origin we have $c=\lambda_{o} \nu_{o}$ while for the measurements on Earth we have $C_{i g}=\lambda_{e} \nu_{e}$.

The second Hubble-Santilli law is then given by

$$
z=\frac{\lambda_{o}}{\lambda_{e}}-1 \approx \pm \frac{\omega_{e}}{\omega_{o}} n_{i g}
$$

where,+- have the same meaning as in law Eq. (28). Even though evidently equivalent to law Eq. (28), the latter formulation allows direct experimental information on intergalactic media, such as its average density geometrically represented by $n_{i g}$, thus allowing contributions to the IRS interpretation of the cosmological redshift due to intergalactic clouds that are independent from teh distance, because characterized by the local medium.

Laws (28) and (30) evidentlyu apply when the relative motion between galaxies is ignorable when compared to the frequency shift caused by the IRS or the IBS. For the case in which possible relativce motions are appreciable, we have from Eq. (13)

$$
z=\frac{\lambda_{o}}{\lambda_{e}}-1 \approx 1 \pm \frac{v_{s}}{V_{\max , s}}+\ldots=1 \pm \frac{v_{s}}{c} \frac{n_{4}}{n_{s}}+\ldots
$$

that, in first approximation identified in Section 2, yields the following:

general Hubble-Santilli law including all possible combinations of frequency shifts due to relative motions with respect to Earth as well as the release or acquisition of energy by light from the medium,

$$
z=\frac{\lambda_{o}}{\lambda_{e}}-1 \approx \pm \frac{v}{c}\left(1 \pm K_{i g} d+\ldots\right)
$$


Note that the Hubble-Santilli laws eliminate the conjecture of "dark matter" because the anomalous redshifts of peripheral galactic stars provide direct experimental information on innergalactic media. Caution is, however, needed to apply law Eq. (26) for the elimination of "dark matter" because the numerical value of $n_{i g}$ is in this case a superposition of the intergalactic and innergalactic index of refractions and the latter evidently depends on the orientation of the galaxies as well as other features.

To understand the cosmological implications of Santilli IRS, one should keep in mind that the HubbleSantilli laws (28), (30) and (32):

1) Have received comprehensive experimental verifications on Earth according to Galileo's teaching, including the verifications presented in this paper;

2) Apply identically to our atmosphere as well as to galaxies, thus implying the validity of the same law throughout the universe, as necessary for physical consistency;

3) They are invariant over time, in the sense of predicting the same numerical values under the same conditions at different time, thanks to the Lorentz-Poincaré-Santilli isosymmetry from which law Eq. (24) is uniquely derived (Sections $1,2,3$ );

4) Eliminate the far reaching conjecture necessary for the Hubble-Doppler law Eq. (23) that a large number of galaxies at the edge of the known universe travel at speed faster than the speed of light in vacuum without any indication on how this could be conceivably possible and what is the origin of the immense energy needed for such speeds;

5) Eliminate the chain of far reaching conjectures on the expansion of the universe, the acceleration of the expansion, the "big bang", the expansion of space itself and, the "dark matter" and the "dark energy".

Needless to say, laws (28), (30) and (32) do not prohibit moderate relative speeds since in this case the full law (14) would apply and their contribution to the cosmological redshift would be ignorable, as it is the case for the contribution due to Earth's rotation for the redshift of the Sun at Sunset or Sunrise studied preceding sections. As a matter of fact, to illustrate the complexity of the universe, Santilli indicates (see, e.g., the review [33]) that, in the eventuality some of the galaxies are made up of antimatter, small local expansions of the universe cannot be ruled out due to the expected gravitational repulsion between matter and antimatter galaxies.

\subsection{LaVillette fit of cosmological redshifts with Zwicky tired light}

During the conduction of these studies, Santilli was unaware of a paper by LaViolette [32] establishing that the fit of cosmological redshift via Zwicky's hypothesis of Tired Light [15] is more accurate than the same fit via the expansion of the universe.

It is then evident that LaViolette's [32] fit of astrophysical data via Zwicky's [15] hypothesis of Tired Light provides an excellent independent backing of Santilli's [9] numerically exact and time invariant representation of the cosmological redshift via the IRS, resulting in the Zwicky-Santilli effect (of course, following the prorating of the average density of Earth's atmosphere to the average density of intergalactic media).

\subsection{Astrophysical implications of Santilli IsoRedShift}

The reader is suggested to meditate a moment on the fact that the representation of the cosmological redshift via the Zwicky-Santilli effect requires the revision of the virtual totality of astrophysical data accumulated during the past century, besides suggesting caution to astrophysicists (and to editors alike) 
before claiming any astrophysical view, such as apparent cosmological blueshift, because the latter could be in reality due to Santilli's IBS (acquisition of energy by light from a hot medium).

Irrespective of the above view, there is no doubt that current cosmological models require structural revisions because, in addition to being disproved by Santilli's measurements on IRS:

1) Each model was ventured in support of the preceding unverifiable model (e.g., the "big bang" conjecture was proposed in support of the expansion of the universe and its acceleration);

2) Said models have reached an excessively conjectural level (such as the conjecture that space itself is expanding in the hope of avoiding Earth at the center of the universe):

3) Said models ultimately imply a violation of special relativity, namely, of the very theory tacitly intended to be maintained (because galaxies at the edge of the known universe must travel at speeds bigger than the speed of light in vacuum due to the need for $v / c>1$ to represent the related cosmological redshifts).

\section{Dismissal of objections against Santilli IsoRedShift}

Following the appearance of measurements [9-14], numerous objections have been raised against Santilli's IRS origin of the redness of the Sun at the horizon. We believe it is important to report these objections, as well as their dismissal, which have been identified following various scientific exchanges.

\subsection{Objection 1. To establish Santilli's IRS for the Sun at the horizon it is necessary to establish it for each individual spectral line}

Dismissal 1. Santilli first conducted the measurements of the IRS for monochromatic laser lines [9] for the specific intent of showing that, when considering the redshift of the Sun, the IRS applies indeed for each individual spectral line. Additionally, as shown in Figs 14 and 15, individual spectral lines of the Sun do indeed experience small IRS for high elevation, which IRS then progressively increases with the decrease of the elevation all the way to shift at the horizon for about $100 \mathrm{~nm}$ per spectral line. In fact, the prorating of the trajectory of the blue laser light in the pipe to the trajectory of Sunlight at the horizon, when prorated to the equivalent trajectory at one atmosphere pressure, yields indeed the $100 \mathrm{~nm}$ of redshift systematically measured to date.

\subsection{Objection 2. All measurements conducted so far do not establish Santilli's IRS for the Sun at the horizon because of insufficient accuracy in the measurement of the intensity of Sunlight}

Dismissal 2. The statement on the insufficient accuracy of Sunlight intensity is correct because the telescope used in the scans has been manually oriented until now per each scan. However, the inaccuracy in the intensity does not apply for Santilli's IRS of Sunlight because variations of the intensity have no effect on the frequency shift as established in the scans of Fig. 31. Alternatively, all spectrometers used in the tests reported in this paper are "wavelength analyzers" (rather than "intensity analyzers"), namely, they are equipment calibrated for the measurements of "wavelengths" (frequencies) and not for Sunlight intensity. In fact, the integration time was selected to have sufficient intensity for the detection of wavelength (frequencies) as indicated in Sections 7, 8. 
10.3. Objection 3. The redness of the Sun at the horizon is partially due to the scattering and partially due to absorption of blue light by our atmosphere, thus leaving red light as predominant at the horizon

Dismissal 3. In the event of scattering of all blue light for the Sun at the horizon, our atmosphere should be blue at sunset and sunrise, while in reality it is red. Also, as clearly established by all scans of Sunlight conducted to date (see Figs 16, 20, etc.), the band of Sunlight surrounding blue completely disappeared in the transition from the Zenith to the horizon and it is shifted for about $100 \mathrm{~nm}$ into the red. Similarly, the band surrounding red is correspondingly shifted into the infrared, also for about $100 \mathrm{~nm}$. Since blue light is known to penetrate deeper than red within transparent media, this experimental evidence establishes that the blue light is shifted all the way to the red in accordance with Santilli's IRS. The remaining part of Objection 3 based on absorption has no physical foundation because red light is known to be absorbed by transparent media faster than blue light, as it is the case in seawater where at the depth of $20 \mathrm{~m}$ only blue light remains visible. In any case, absorption of Sunlight is set by the chemistry of our atmosphere. Therefore, the only possibility of having a frequency shift via absorption would be to change the entire Earth's atmosphere.

\subsection{Objection 4. The redness of the Sun at Sunset is due to Rayleigh and other scattering (see, e.g., Refs. [26-31]) and it is not due to Santilli's IRS}

Dismissal 4. Rayleigh scattering was developed (and never experimentally verified to our knowledge) for the color of the atmosphere which is manifestly due to scattering of Sunlight (otherwise our sky would be black day and night). By its own derivation, Rayleigh "scattering" cannot be consistently applied to direct Sunlight reaching us along a straight line at the horizon (see footnote ${ }^{3}$ ). Rayleigh and other scatterings are indeed applicable for the representation of the "intensity" of that percentage of Sunlight which is lost from our direct view because of scattering in air that disperses Sunlight in all directions. Independently from all that, Rayleigh and other scattering provide a phenomenological description without any representation of the physical origin of the frequency shift. By contrast, Santilli's IRS is centered in the physical origin of the frequency shift, loss of energy by all frequencies of light to air, in whose framework intensities is merely used to achieve measurable wavelengths. Also, one century of astrophysical knowledge has been based on the fundamental measurement of cosmological shift of "frequencies" and complemented with measurements of the "intensity" (luminosity) of far away stars and galaxies. Dismissing for Earth's measurements the same complementarity of Santilli's "frequency" IRS with any desired representation of the "intensity" variation would be a double standard, particularly in view of the fact that Santilli's IRS recovers identically Doppler's shift when moving from a physical media to the vacuum.

\subsection{Objection 5. The measurements of Santilli's IRS are wrong because they violate special relativity due to Sunlight non-conservation of energy and momentum}

Dismissal 5. The Minkowski line element is known to be symmetric, that is, holding for both forward and backward in time, due to its quadratic character in the variables, Eq. (7). Consequently, the very axioms of special relativity solely apply for systems reversible over time, such as atomic structures, particles in accelerators, elastic scatterings, and many more systems. However, clear experimental evidence visible to our naked eye establishes that events in our environment are irreversible over time and 
cannot be consistently reduced to a finite number of reversible elementary particles, as known by qualified experts. Therefore, the dismissal of Santilli's IRS just because it is based on non-conservation of Sunlight energy and momentum implies the nonscientific aim of adapting physical reality to a preferred theory, rather than modifying the theory to represent physical reality. In any case, Santilli has shown that Sunlight non-conservation of energy and momentum solely applies for the 20th century formulation of special relativity, and does not apply for the broader isotopic realization of the same axioms, as shown in Section 3. Therefore, Objection 6 is invalid, firstly, because physical reality is nonconservative and, secondly, because the axioms of special relativity are not violated under Sunlight non-conservation of energy and momentum. Finally, as stated in section three, it is rather remarkable that the Minkowski line Eq. (7) element is quadratic in its variables, while its isotopic covering Eq. (9) is not necessarily quadratic due to the dependence of the characteristic $n$ - quantities in the local variable. Therefore, Santilli's isotopies of special relativity enlarge the applicability of its axioms to nonconservative and irreversible systems - precisely as needed to represent the IRS.

10.6. Objection 6. The measurements of Santilli's IRS in Earth's atmosphere, once applied to the representation of the cosmological redshifts, yield very large deviations from astrophysical values, thus having no cosmological relevance

Dismissal 6. As established by experimental evidence (see, e.g., Fig. 5), Santilli's IRS is proportional to the density of the medium. Therefore, the reduction of the average density of our atmosphere to that of intergalactic media implies an evident large decrease of numerical values, since the latter is dramatically more rarefied than the former. Hence, Objection 6 has no physical value. Independently from that, once interpreted via Santilli's IRS, the numerical value of the cosmological redshift provides direct experimental information on the intergalactic medium.

\subsection{Objection 7. Santilli's IRS cannot represent cosmological redshifts because, in the event photons lose energy to the intergalactic medium, the images of distant galaxies should be blurred, yet no such blurring has been detected}

Dismissal 7. Santilli's measurements of direct Sunlight and monochromatic laser light shifts are based on a loss of energy due to the interaction of electromagnetic waves with matter without any scattering, thus representing the cosmological redshift without any blurriness. Scattering of galactic lights does indeed occur, but it is responsible for the decrease of the intensity of the galactic light, exactly as it occurs for direct Sunlight at the horizon.

\subsection{Objection 8. It is not true that the expansion of the universe implies Earth at the center of the universe because the same expansion is visible anywhere throughout the universe}

Dismissal 8. Objection 8 would be valid in the event the expansion of the universe were uniform. The objection is invalidated by the acceleration of the expansion according to which, according to Hubble's law and one century of measurements, the cosmological redshift is proportional to the distance from Earth, thus being interpreted via the acceleration of the expansion, radially from Earth in all directions in space. Such a geometry of the claimed acceleration of the expansion clearly mandates Earth being at the center of the universe (see Figs 32 and 33). The same conclusion is also implied by the big bang due to its claim of representing the radial distributions from Earth of all galaxies. The far reaching conjecture of the expansion of space itself was ventured precisely to avoid Earth being at the center of the universe. 
However, the experimental evidence on the radial acceleration of the expansion from Earth in all space directions implies, again, that Earth is at the center of the universe despite the conjectural hypothesis that space itself is expanding.

\subsection{Objection 9. It is not true that galaxies at the edge of the universe violate special relativity because in this case general relativity is applicable and known coordinate transformations reduce the speed of galaxies to values $v<c$}

Dismissal 9. Astrophysical evidence establishes beyond doubt that, according to current cosmological models, the center of galaxies accelerates along a straight radial line from Earth, thus prohibiting the meaningful use of curvature. Also, intergalactic gravitational attractions are so small to be ignorable, thus preventing any sound use of general relativity, thus leaving special relativity the sole truly applicable discipline. Additionally, the same coordinate transformations mentioned in the objection could be equally used to turn speed $v<c$ of near by galaxies into values $v>c$, by therefore mandating the sole use for all astrophysical measurements of the actual reference frame of the observer on Earth.

The authors would gratefully appreciate the communication of any additional objections against Santilli's IRS that are not reported above.

\section{Concluding remarks}

In the authors' view, the most important cosmological implication of Santilli's mathematical, theoretical and experimental research on anomalous frequency shifts of electromagnetic waves propagating within physical, is the termination of the dominance of Einstein special relativity for the large scale structure of the universe, thus suggesting the advent of suitable covering theories along the New Sciences for a New Era of Ref. [33]. ${ }^{7}$

More generally, the measurements presented in this paper confirm Santilli's fifty years of research on the inapplicability (rather than the violation) of special relativity for all interior dynamical problems of extended particles and electromagnetic waves propagating within physical media, in favor of Santilli's central methodological notion of mutation of spacetime caused by the presence of matter (or energy), with ensuing validity of the covering Lorentz-Poincaré-Santilli isosymmetry and related isorelativity as outlined in Sections 1, 2, 3 .

This occurrence will inevitably require a re-inspection of the virtual entire 20th century physics representing interior dynamical problems in a form compatible with Einstein special relativity, thus including a re-inspection of the structure of hadrons, nuclei, stars, high energy scattering theories, grand unified theories including the structure (rather than the sole classification) of particles, and other fields.

It is appropriate to recall here Santilli's insistence that particles can indeed have "point-like charges" like the electron, but there exist no particles with "point-like wavepackets". This implies that hadrons,

\footnotetext{
${ }^{7}$ Einstein's general relativity has never been plausibly applicable to the large scale structure of the universe due to the large intergalactic distances, with consequential virtually null, large scale Riemannian curvature. At any rate, Einstein's gravitation has remained afflicted by very serious consistency problems that have remained unresolved for a century due to the general dismissal of their identification in refereed journals (see, e.g., the introductory outline by R. Anderson at www.santillifoundation.org/Isogravitation.php). There is little doubt that, with a virtually null intergalactic gravitational field, as well as with unresolved fundamental structural problems, large scale studies on the structure of the universe based on Einstein gravitation will not resist the test of time.
} 
nuclei, stars and scattering regions are composed by a hyperdense medium characterized by wavepacksts under conditions of total mutual penetration, rather than by isolated point particles as currently conjectured.

Therefore, the experimental evidence confirmed in this paper on deviations from special relativity within a medium of such a light density as Earth's atmosphere, establishes the inapplicability of special relativity within hadrons, nuclei, stars and scattering regions beyond a scientific doubt.

Independently from the above, to seriously understand and appraise the scientific moment, the reader should know that Santilli's mutation of spacetime has received experimental confirmations in numerous interior dynamical conditions ranging from classical mechanics to biology (see Ref. [33] for a general review).

As Santilli puts it: "Rather than abusing Einstein's name by applying the 20th century realization of his theories under conditions they were never intended for and are experimentally disproved in any case, the best way to honor Einstein's name is that of maintaining his axioms, and merely introducing broader realizations for the representation of more complex physical conditions as permitted by the very conception and technical realization of the broader isomathematics."

\section{Note added}

Following the completion of this paper, the authors have been informed of the appearance of the following publications significant for the studies herein presented:

1) The content of Section 3 on the axiomatic compatibility of the IsoRedShift with special relativity is being studied in detail in the paper by R. M. Santilli. "Apparent compatibility of super/subluminal speeds of electromagnetic waves with special relativity axioms," IBR preprint 033th-12, http://www.scientificethics.org/super-sub-luminal-speeds.pdf.

2) The sole validity of Rayleight et al. scattering models for Earth's atmosphere, rather than direct Sunlight, has been studied in details via comments on I. Gandzha's Ref. [31] in the paper by R. Cox, "Comments on I. Gandzha's Paper: "Solar Redshift Calculation by the Rayleigh Scattering Mechanism," Hadronic J. Vol. 35 (2012), in press, http://www.scientificethics.org/CriticismsGandzha.pdf, which presents a comprehensive analysis of:

A) The impossibility for the Rayleigh "scattering" to provide an explanation of the origin as well as a complete description of the redshift of "direct" Sunlight at the horizon;

B) The impossibility of using the measurements presented in this paper on "frequencies" as experimental confirmation of the behavior of Rayleigh "intensities;" and

C) The complementarity of Rayleigh scattering and Santilli IsoRedShift, provided that the latter is used for the origin and behavior of frequencies of the portion of direct Sunlight reaching us (as verified in this paper), while the former is used for the origin and behavior of the intensities of the part of direct Sunlight which is absorbed by our atmosphere due to scattering according to the original conception by Rayleigh.

3) Astrophysical evidence on the absence of the expansion of the universe, completely independent from Santilli's IsoRedShift, yet confirming and complementing the latter, has been studied in the paper by Eric J. Lerner, "Evidence for a Non-Expanding Universe: Surface Brightness Data From HUDF," arXiv:astro-ph/050961. For the reader's convenience, we reproduce here the abstract of the above paper due to the evident compatibility and complementary of Lermner's astrophysical studies with Santilli's IsoRedShift: 
Abstract. Surface brightness data can distinguish between a Friedman-Robertson-Walker expanding universe and a nonexpanding universe. For surface brightness measured in $\mathrm{AB}$ magnitudes per angular area, all FRW models, regardless of cosmological parameters, predict that surface brightness declines with redshift as $(z+1)-3$, while any non-expanding model predicts that surface brightness is constant with distance and thus with z. High-z UV surface brightness data for galaxies from the Hubble Ultra Deep Field and low-Z data from GALEX are used to test the predictions of these two models up to $z=6$. A preliminary analysis presented here of samples observed at the same at-galaxy wavelengths in the UV shows that surface brightness is constant, $m=k z 0.026+0.15$, consistent with the nonexpanding model. This relationship holds if distance is linearly proportional to $z$ at all redshifts, but seems insensitive to the particular choice of d-z relationship. Attempts to reconcile the data with FRW predictions by assuming that high-z galaxies have intrinsically higher surface brightness than low-Z galaxies appear to face insurmountable problems. The intrinsic FUV surface brightness required by the FRW models for high-z galaxies exceeds the maximum FUV surface brightness of any low-z galaxy by as much as a factor of 40. Dust absorption appears to make such extremely high intrinsic FUV surface brightness physically impossible. If confirmed by further analysis, the impossibility of such high-m galaxies would rule out all FRW expanding universe (big bang) models.

\section{Acknowledgments}

The authors would like to thank Prof. R. M. Santilli for invaluable assistance in the drafting and finalization of this paper. Thanks is also due to all participants of the 2012 Seminar Course in Hadronic Mathematics, Mechanics and Chemistry, held at the island of Kos, Greece, from September 9 to 25, 2012, for their in-depth discussions and analyses of the data reported in this paper. Particular thanks are due to Mrs. D. Zuckerman for a detailed linguistic control of the entire manuscript, although the authors are solely responsible for its content due to various final touches.

\section{References}

[1] R.M. Santilli, Hadronic J 1 (1978), 223, http://www.santilli-foundation.org/docs/Santilli-58.pdf.

[2] R.M. Santilli, Foundation of Theoretical Mechanics, I (1978), [2a], and Volume II (1982) [2b], Springer-Verlag, Heidelberg, Germany, http://www.santilli-foundation.org/docs/Santilli-209.pdf, http://www.santilli-foundation.org/docs/ santilli-69.pdf.

[3] R.M. Santilli, Nonlocal-Integral Isotopies of Differential Calculus, Mechanics and Geometries, in Isotopies of Contemporary Mathematical Structures, P. Vetro Editor, Rendiconti Circolo Matematico Palermo, Suppl 42 (1996), 7-82, http://www.santilli-foundation.org/docs/Santilli-37.pdf.

[4] D.S. Sourlas and Gr.T. Tsagas, Mathematical Foundation of the Lie-Santilli Theory, Ukraine Academy of Sciences (1993), http://www.santilli-foundation.org/docs/santilli-70.pdf.

[5] R.M. Santilli, Isominkowskian geometry for the gravitaitonal treatment of matter and its isodual for antimatter, Intern $J$ Modern Phys $D 7$ (1998), 351, http://www.santilli-foundation.org/docs/Santilli-35.pdf.

[6] R.M. Santilli, Lie-isotopic lifting of special relativity for extended deformable particles, Lettere Nuovo Cimento 37 (1983), 545, http://www.santilli-foundation.org/docs/Santilli-50.pdf.

[7] R.M. Santilli, Isotopic Generalizations of Galilei and Einstein Relativities, I [7a] and II [7b] (1991), International Academic Press, http://www.santilli-foundation.org/docs/Santilli-01.pdf, http://www.santilli-foundation.org/docs/Santilli61.pdf.

[8] H. Arp, Quasars Redshift and Controversies, Interstellar Media, Berkeley (1987).

[9] R.M. Santilli, Experimental Verifications of IsoRedShift with Possible Absence of Universe Expansion, Big Bang, Dark Matter, and Dark Energy, The Open Astronomy Journal 3 (2010), 124, http://www.santilli-foundation.org/docs/Santilliisoredshift.pdf. 
[10] R.M. Santilli, Experimental Verification of IsoRedShift and its Cosmological Implications, American Institute of Physics Conference Proceedings 1281 (2010), 882-885, http://www.santilli-foundation.org/docs/Isoredshift-Letter.pdf.

[11] R.M. Santilli, Iso-Minkowskian Geometry for Interior Dynamical Problems, Contributed paper to Cosmology, Quantum Vacuum, and Zeta Functions, Diego Sez-Gmez Sergei Odintsov Sebasti Xamb Editors, Springer, 2011, http://www. santilli-foundation.org/docs/Isominkowskian-Geom.pdf.

[12] G. West and G. Amato, Experimental Confirmation of Santilli's IsoRedShift and IsoBlueShift, contributed paper to the Proceedings of the San Marino Workshop on Astrophysics and Cosmology for Matter and Antimatter, Republic of San Marino, September 5 to 9, 2011, in press (2012).

[13] R. Verreault, Experimental confirmation of Santilli's prediction of the anisotropy of Earth's gravitational field via the spherical pendulum, Journal of Computational Methods in Sciences and Engineering, 2012, in press.

[14] R.M. Santilli, G. Amato and G. West, Experimental Confirmation of the IsoRedShift at Sun at Sunset and Sunrise with Consequential Absence of Universe Expansion and Related Conjectures, Journal of Computational Methods in Sciences and Engineering 12 (2012), 165-188, http://www.santilli-foundation.org/docs/Confirmation-sun-IRS.pdf.

[15] F. Zwicky, On the Red Shift of Spectral Lines through Interstellar Space, Proceedings of the National Academy of Sciences of the United States of America, 15 (1929), 773, http://www.pnas.org/cgi/reprintframed/15/10/773.

[16] C.W. Misner, K.S. Thorne and J.A. Wheeler, Gravitation, W. H. Feeman and Company (1973).

[17] P. Riess et al., Astronomical Journal 116 1998), 1009; S. Perlmutter et al., Astrophysical Journal 517 (1999), 565.

[18] E.J. Wollack, Edward, The Study of the Universe: Big Bang Theory, NASA (2010).

[19] A.B. Whiting, The expansion of space: free-particle motion and the cosmological redshift, The Observatory 124 (2004), 174, http://adsabs.harvard.edu/abs/2004Obs...124174W.

[20] E. Hubble, Proceedings of the national academy of sciences of the united states of america, 15 (1929), 168.

[21] V. Rubin, Rotation of the andromeda nebula from a spectroscopic survey of emission regions, Astrophysical Journal 159 (1970), 379, http://adsabs.harvard.edu/doi/10.1086/150317.

[22] C. Corda, H.J. Mosquera-Questa, R. Lorduy and G. Homez, High energy R2 gravity as a model for dark matter in galaxies, Astroparticle Physics 35 (2012), 362, www.sciencedirect.com/science/article/pii/S0927650511001678.

[23] P.J. Peebles and B. Ratra, The cosmological constant and dark energy, Reviews of Modern Physics 75 (2003, 559 , http://adsabs.harvard.edu/abs/2003RvMP...75..559P.

[24] H. Ahmar, G. Amato, S. Moskaliuk, J.V. Kadeisvili, P. LaViolette, J. Manuel and G. West, Raw data on the IRS measurements of Sunlight from the Zenith to Sunset obtained in the island of Kos, Greece, on 9/20/12, http://www.santillifoundation.org/docs/Kos-Greece-Data.zip.

[25] H. Ahmar, G. Amato, S. Moskaliuk, J.V. Kadeisvili, P. LaViolette, J. Manuel and G. West, Raw data on the IRS measurements of Sunlight from the Sunrise to the Zenith obtained at Cocoa Beach, West Florida, on 10/20/12, http://www.santillifoundation.org/docs/Cocoa-Beach-data.zip.

[26] J.S. Rayleigh, On the light from the sky, its polarization and colour, Philosophical Magazine, series 4, 41 1871, pp. 107120, 274279;

[27] J. Strutt, On the scattering of light by small particles, Philosophical Magazine series 4, 41 (1871), 447-454.

[28] E. Schoenberg, Handbuch der Astrophysik. Band II, erste HŁfte. Berlin: Springer, 1929.

[29] G. Busch and R. Verreault, A new method to demonstrate and measure birefrangence, Acta Cryst. A 27 (1971), 680.

[30] C. Gueymard and H. Kambezidis, Solar Spectral Radiation (Chapter 5 in Solar Radiation and Daylight Models, ) Muneer T., Elsevier, 2004.

[31] I. Gandzha, Solar redshift calculations via the Rayleight scattering mechanism, in press at the Hadronic Journal 35 (2012).

[32] LaViolette, Paul A. Is the universe really expanding? The Astrophysical Journal 301 (1986), 544-553.

[33] I. Gandzha and J. Kadeisvili, New Sciences for a New Era: Mathematical, Physical and Chemical Discoveries of Ruggero Maria Santilli, Sankata Printing Press, Nepal (2011), http://www.santilli-foundation.org/docs/RMS.pdf. 